TRANSACTIONS OF THE

AMERICAN MATHEMATICAL SOCIETY

Volume 361, Number 4, April 2009, Pages 2085-2107

S 0002-9947(08)04767-3

Article electronically published on November 5, 2008

\title{
THE COKERNEL OF THE JOHNSON HOMOMORPHISMS OF THE AUTOMORPHISM GROUP OF A FREE METABELIAN GROUP
}

\author{
TAKAO SATOH
}

Dedicated to Professor Shigeyuki Morita on the occasion of his 60th birthday

\begin{abstract}
In this paper, we determine the cokernel of the $k$-th Johnson homomorphisms of the automorphism group of a free metabelian group for $k \geq 2$ and $n \geq 4$. As a corollary, we obtain a lower bound on the rank of the graded quotient of the Johnson filtration of the automorphism group of a free group. Furthermore, by using the second Johnson homomorphism, we determine the image of the cup product map in the rational second cohomology group of the IA-automorphism group of a free metabelian group, and show that it is isomorphic to that of the IA-automorphism group of a free group which is already determined by Pettet. Finally, by considering the kernel of the Magnus representations of the automorphism group of a free group and a free metabelian group, we show that there are non-trivial rational second cohomology classes of the IA-automorphism group of a free metabelian group which are not in the image of the cup product map.
\end{abstract}

\section{INTRODUCTION}

Let $G$ be a group and $\Gamma_{G}(1)=G, \Gamma_{G}(2), \ldots$ its lower central series. We denote by Aut $G$ the group of automorphisms of $G$. For each $k \geq 0$, let $\mathcal{A}_{G}(k)$ be the group of automorphisms of $G$ which induce the identity on the quotient group $G / \Gamma_{G}(k+1)$. Then we obtain a descending central filtration

$$
\text { Aut } G=\mathcal{A}_{G}(0) \supset \mathcal{A}_{G}(1) \supset \mathcal{A}_{G}(2) \supset \cdots
$$

of Aut $G$, called the Johnson filtration of Aut $G$. This filtration was introduced in 1963 with a pioneer work by S. Andreadakis [1]. For each $k \geq 1$, set $\mathcal{L}_{G}(k):=$ $\Gamma_{G}(k) / \Gamma_{G}(k+1)$ and $\operatorname{gr}^{k}\left(\mathcal{A}_{G}\right)=\mathcal{A}_{G}(k) / \mathcal{A}_{G}(k+1)$. Let $G^{\text {ab }}$ be the abelianization of $G$. Then, for each $k \geq 1$, an Aut $G^{\text {ab }}$-equivariant injective homomorphim

$$
\tau_{k}: \operatorname{gr}^{k}\left(\mathcal{A}_{G}\right) \rightarrow \operatorname{Hom}_{\mathbf{Z}}\left(G^{\mathrm{ab}}, \mathcal{L}_{G}(k+1)\right)
$$

is defined. (For a definition, see Subsection 2.1.2.) This is called the $k$-th Johnson homomorphism of Aut $G$. Historically, the study of the Johnson homomorphism was begun in 1980 by D. Johnson [17. He studied the Johnson homomorphism of a mapping class group of a closed oriented surface, and determined the abelianization

Received by the editors May 17, 2007.

2000 Mathematics Subject Classification. Primary 20F28; Secondary 20J06.

Key words and phrases. Automorphism group of a free metabelian group, Johnson homomorphism, second cohomology group, Magnus representation. 
of the Torelli group. (See [18.) There is a broad range of remarkable results for the Johnson homomorphisms of a mapping class group. (For example, see [14] and [25.)

Let $F_{n}$ be a free group of rank $n$ with basis $x_{1}, \ldots, x_{n}$ and $F_{n}^{M}$ the free metabelian group of rank $n$. Namely $F_{n}^{M}$ is the quotient group of $F_{n}$ by the second derived series $\left[\left[F_{n}, F_{n}\right],\left[F_{n}, F_{n}\right]\right]$ of $F_{n}$. Then both abelianizations of $F_{n}$ and $F_{n}^{M}$ are a free abelian group of rank $n$, denoted by $H$. Fixing a basis of $H$ induced from $x_{1}, \ldots, x_{n}$, we can identify Aut $G^{\text {ab }}$ with $\operatorname{GL}(n, \mathbf{Z})$ for $G=F_{n}$ and $F_{n}^{M}$. For simplicity, throughout this paper we write $\Gamma_{n}(k), \mathcal{L}_{n}(k), \mathcal{A}_{n}(k)$ and $\operatorname{gr}^{k}\left(\mathcal{A}_{n}\right)$ for $\Gamma_{F_{n}}(k), \mathcal{L}_{F_{n}}(k), \mathcal{A}_{F_{n}}(k)$ and $\operatorname{gr}^{k}\left(\mathcal{A}_{F_{n}}\right)$ respectively. Similarly, we write $\Gamma_{n}^{M}(k), \mathcal{L}_{n}^{M}(k), \mathcal{A}_{n}^{M}(k)$ and $\operatorname{gr}^{k}\left(\mathcal{A}_{n}^{M}\right)$ for $\Gamma_{F_{n}^{M}}(k), \mathcal{L}_{F_{n}^{M}}(k), \mathcal{A}_{F_{n}^{M}}(k)$ and $\operatorname{gr}^{k}\left(\mathcal{A}_{F_{n}^{M}}\right)$ respectively. The first aim of this paper is to determine the $\mathrm{GL}(n, \mathbf{Z})$-module structure of the cokernel of the Johnson homomorphisms $\tau_{k}$ of Aut $F_{n}^{M}$ for $n \geq 4$ as follows:

Theorem 1. For $k \geq 2$ and $n \geq 4$,

$$
0 \rightarrow \operatorname{gr}^{k}\left(\mathcal{A}_{n}^{M}\right) \stackrel{\tau_{k}}{\longrightarrow} H^{*} \otimes_{\mathbf{Z}} \mathcal{L}_{n}^{M}(k+1) \stackrel{\operatorname{Tr}_{k}^{M}}{\longrightarrow} S^{k} H \rightarrow 0
$$

is a $\mathrm{GL}(n, \mathbf{Z})$-equivariant exact sequence.

Here $S^{k} H$ is the symmetric product of $H$ of degree $k$, and $\operatorname{Tr}_{k}^{M}$ is a certain $\mathrm{GL}(n, \mathbf{Z})$-equivariant homomorphism called the Morita trace introdued by S. Morita 24]. (For definition, see Subsection 3.2.)

From Theorem 1, we can give a lower bound on the rank of $\operatorname{gr}^{k}\left(\mathcal{A}_{n}\right)$ for $k \geq 2$ and $n \geq 4$. The study of the Johnson filtration of Aut $F_{n}$ was begun in the 1960s by Andreadakis [1] who showed that for each $k \geq 1$ and $n \geq 2, \operatorname{gr}^{k}\left(\mathcal{A}_{n}\right)$ is a free abelian group of finite rank, and that $\mathcal{A}_{2}(k)$ coincides with the $k$-th lower central series of the inner automorphism group Inn $F_{2}$ of $F_{2}$. Furthermore, he [1] computed $\operatorname{rank}_{\mathbf{Z}} \operatorname{gr}^{k}\left(\mathcal{A}_{2}\right)$ for all $k \geq 1$. However, the structure of $\operatorname{gr}^{k}\left(\mathcal{A}_{n}\right)$ for general $k \geq 2$ and $n \geq 3$ is much more complicated. Set $\tau_{k, \mathbf{Q}}=\tau_{k} \otimes \mathrm{id}_{\mathbf{Q}}$, and call it the $k$-th rational Johnson homomorphism. For any $\mathbf{Z}$-module $M$, we denote $M \otimes_{\mathbf{Z}} \mathbf{Q}$ by the symbol obtained by attaching a subscript $\mathbf{Q}$ to $M$, like $M_{\mathbf{Q}}$ and $M^{\mathbf{Q}}$. For $n \geq 3$, the $\mathrm{GL}(n, \mathbf{Z})$-module structure of $\operatorname{gr}_{\mathbf{Q}}^{2}\left(\mathcal{A}_{n}\right)$ is completely determined by Pettet [31. In our previous paper [33, we determined those of $\operatorname{gr}_{\mathbf{Q}}^{3}\left(\mathcal{A}_{n}\right)$ for $n \geq 3$. For $k \geq 4$, the $\operatorname{GL}(n, \mathbf{Z})$-module structure of $\operatorname{gr}_{\mathbf{Q}}^{k}\left(\mathcal{A}_{n}\right)$ is not determined. Furthermore, even its dimension is also unknown.

Let $\nu_{n}$ : Aut $F_{n} \rightarrow$ Aut $F_{n}^{M}$ be a natural homomorphism induced from the action of Aut $F_{n}$ on $F_{n}^{M}$. By notable works due to Bachmuth and Mochizuki [5], it is known that $\nu_{n}$ is surjective for $n \geq 4$. They [4] also showed that $\nu_{3}$ is not surjective. In Subsection 3.1 we see that the homomorphism $\bar{\nu}_{n, k}: \operatorname{gr}^{k}\left(\mathcal{A}_{n}\right) \rightarrow \operatorname{gr}^{k}\left(\mathcal{A}_{n}^{M}\right)$ induced from $\nu_{n}$ is also surjective for $n \geq 4$. Hence we have

Corollary 1. For $k \geq 2$ and $n \geq 4$,

$$
\operatorname{rank}_{\mathbf{Z}}\left(\operatorname{gr}^{k}\left(\mathcal{A}_{n}\right)\right) \geq n k\left(\begin{array}{c}
n+k-1 \\
k+1
\end{array}\right)-\left(\begin{array}{c}
n+k-1 \\
k
\end{array}\right) .
$$

We should remark that in general, equality does not hold, since for instance $\operatorname{rank}_{\mathbf{z}} \operatorname{gr}^{3}\left(\mathcal{A}_{n}\right)=n\left(3 n^{4}-7 n^{2}-8\right) / 12$, which is not equal to the right hand side of the inequality above.

Next, we consider the second cohomology group of the IA-automorphism group of the free metabelian group. Here the IA-automorphism group $\mathrm{IA}(G)$ of a group $G$ 
is defined to be a group which consists of automorphisms of $G$ which trivially act on the abelianization of $G$. By the definition, $\mathrm{IA}(G)=\mathcal{A}_{G}(1)$. We write $\mathrm{IA}_{n}$ and $\mathrm{IA}_{n}^{M}$ for $\operatorname{IA}\left(F_{n}\right)$ and $\operatorname{IA}\left(F_{n}^{M}\right)$ for simplicity. Let $H^{*}:=\operatorname{Hom}_{\mathbf{Z}}(H, \mathbf{Z})$ be the dual group of $H$. Then we see that the first homology group of $\mathrm{IA}_{n}^{M}$ for $n \geq 4$ is isomorphic to $H^{*} \otimes_{\mathbf{z}} \Lambda^{2} H$ in the following way. Let $\nu_{n, 1}: \mathrm{IA}_{n} \rightarrow \mathrm{IA}_{n}^{M}$ be the restriction of $\nu_{n}$ to $\mathrm{IA}_{n}$. Bachmuth and Mochizuki [5] showed that $\nu_{n, 1}$ is surjective for $n \geq 4$. This fact sharply contrasts with their previous work [4, which shows there are infinitely many automorphisms of $\mathrm{IA}_{3}^{M}$ which are not contained the image of $\nu_{3,1}$. On the other hand, by independent works of Cohen-Pakianathan [9, 10, Farb [11] and Kawazumi [19], $H_{1}\left(\mathrm{IA}_{n}, \mathbf{Z}\right) \cong H^{*} \otimes_{\mathbf{z}} \Lambda^{2} H$ for $n \geq 3$. Since the kernel of $\nu_{n, 1}$ is contained in the commutator subgroup of $\mathrm{IA}_{n}^{M}$, we have $H_{1}\left(\mathrm{IA}_{n}^{M}, \mathbf{Z}\right) \cong H^{*} \otimes \mathbf{z} \Lambda^{2} H$ for $n \geq 4$. (See Subsection 2.3.) In general, however, there are few results for computation of the (co)homology groups of $\mathrm{IA}_{n}^{M}$ of higher dimensions. In this paper we determine the image of the cup product map in the rational second cohomology group of $\mathrm{IA}_{n}^{M}$, and show that it is isomorphic to that of $\mathrm{IA}_{n}$, using the second Johnson homomorphism. Namely, let $\cup_{\mathbf{Q}}: \Lambda^{2} H^{1}\left(\mathrm{IA}_{n}, \mathbf{Q}\right) \rightarrow H^{2}\left(\mathrm{IA}_{n}, \mathbf{Q}\right)$ and $\cup_{\mathbf{Q}}^{M}: \Lambda^{2} H^{1}\left(\mathrm{IA}_{n}^{M}, \mathbf{Q}\right) \rightarrow H^{2}\left(\mathrm{IA}_{n}^{M}, \mathbf{Q}\right)$ be the rational cup product maps of $\mathrm{IA}_{n}$ and $\mathrm{IA}_{n}^{M}$ respectively. In Subsection 4.2, we show

Theorem 2. For $n \geq 4, \nu_{n, 1}^{*}: \operatorname{Im}\left(\cup_{\mathbf{Q}}^{M}\right) \rightarrow \operatorname{Im}\left(\cup_{\mathbf{Q}}\right)$ is an isomorphism.

Here we should remark that the $\operatorname{GL}(n, \mathbf{Z})$-module structure of $\operatorname{Im}\left(\cup_{\mathbf{Q}}\right)$ is completely determined by Pettet [31] for any $n \geq 3$.

Now, for the study of the second cohomology group of $\mathrm{IA}_{n}^{M}$, it is also an important problem to determine whether the cup product map $\cup_{\mathbf{Q}}^{M}$ is surjective or not. For the case of $\mathrm{IA}_{n}$, it is still not known whether $\cup_{\mathbf{Q}}$ is surjective or not. In the last section, we prove that the rational cup product map $\cup_{\mathbf{Q}}^{M}$ is not surjective for $n \geq 4$. It is easily seen that $\mathcal{K}_{n}$ is an infinite subgroup of $\mathrm{IA}_{n}$, since $\mathcal{K}_{n}$ contains the second derived series of the inner automorphism group of a free group $F_{n}$. The structure of $\mathcal{K}_{n}$ is, however, very complicated. For example (finitely or infinitely many) generators and the abelianization of $\mathcal{K}_{n}$ are still not known. To clarify the structure of $\mathcal{K}_{n}$, it is also important to study the obstruction for the faithfulness of the Magnus representation of $\mathrm{IA}_{n}$ since $\mathcal{K}_{n}$ is equal to the kernel, by a result of Bachmuth 2]. (See Subsection 2.3])

From the cohomological five-term exact sequence of the group extension

$$
1 \rightarrow \mathcal{K}_{n} \rightarrow \mathrm{IA}_{n} \rightarrow \mathrm{IA}_{n}^{M} \rightarrow 1,
$$

it suffices to show the non-triviality of $H^{1}\left(\mathcal{K}_{n}, \mathbf{Q}\right)^{\mathrm{IA}_{n}}$ to show that $\operatorname{Im}\left(\cup_{\mathbf{Q}}^{M}\right) \neq$ $H^{2}\left(\mathrm{IA}_{n}^{M}, \mathbf{Q}\right)$. Set $\overline{\mathcal{K}}_{n}:=\mathcal{K}_{n} /\left(\mathcal{K}_{n} \cap \mathcal{A}_{n}(4)\right) \subset \operatorname{gr}^{3}\left(\mathcal{A}_{n}\right)$. Then $\overline{\mathcal{K}}_{n}$ naturally has a $\operatorname{GL}(n, \mathbf{Z})$-module structure, and the natural projection $\mathcal{K}_{n} \rightarrow \overline{\mathcal{K}}_{n}$ induces an injective homomorphism $H^{1}\left(\overline{\mathcal{K}}_{n}, \mathbf{Q}\right) \rightarrow H^{1}\left(\mathcal{K}_{n}, \mathbf{Q}\right)^{\mathrm{IA}_{n}}$. In this paper, we determine the $\mathrm{GL}(n, \mathbf{Z})$-module structure of $H_{1}\left(\overline{\mathcal{K}}_{n}, \mathbf{Q}\right)$ using the rational third Johnson homomorphism of Aut $F_{n}$. The non-triviality of $H^{1}\left(\overline{\mathcal{K}}_{n}, \mathbf{Q}\right)$ immediately follows from it. In Subsection 5.1, we show

Theorem 3. For $n \geq 4, \tau_{3, \mathbf{Q}}\left(\overline{\mathcal{K}}_{n}^{\mathbf{Q}}\right) \cong H_{\mathbf{Q}}^{[2,1]} \oplus\left(D \otimes_{\mathbf{Q}} H_{\mathbf{Q}}^{\left[3,2^{2}, 1^{n-4}\right]}\right)$.

Here $H^{\lambda}$ denotes the Schur-Weyl module of $H$ corresponding to the Young diagram $\lambda=\left[\lambda_{1}, \ldots, \lambda_{l}\right]$, and $D:=\Lambda^{n} H$ the one-dimensional representation of 
$\mathrm{GL}(n, \mathbf{Z})$ given by the determinant map. Since $\tau_{3, \mathbf{Q}}$ is injective, this shows that

$$
\overline{\mathcal{K}}_{n}^{\mathbf{Q}} \cong H_{\mathbf{Q}}^{[2,1]} \oplus\left(D \otimes \mathbf{Q} H_{\mathbf{Q}}^{\left[3,2^{2}, 1^{n-4}\right]}\right) .
$$

As a corollary, we have

Corollary 2. For $n \geq 4$,

$$
\operatorname{rank}_{\mathbf{Z}}\left(H_{1}\left(\mathcal{K}_{n}, \mathbf{Z}\right)\right) \geq \frac{1}{3} n\left(n^{2}-1\right)+\frac{1}{8} n^{2}(n-1)(n+2)(n-3) .
$$

Finally, we obtain

Theorem 4 . For $n \geq 4$, the rational cup product

$$
\cup_{\mathbf{Q}}^{M}: \Lambda^{2} H^{1}\left(\mathrm{IA}_{n}^{M}, \mathbf{Q}\right) \rightarrow H^{2}\left(\mathrm{IA}_{n}^{M}, \mathbf{Q}\right)
$$

is not surjective, and

$$
\operatorname{dim}_{\mathbf{Q}}\left(H^{2}\left(\mathrm{IA}_{n}^{M}, \mathbf{Q}\right)\right) \geq \frac{1}{24} n(n-2)\left(3 n^{4}+3 n^{3}-5 n^{2}-23 n-2\right) .
$$

In Section 2. we recall the IA-automorphism group of $G$ and the Johnson homomorphisms of the automorphism group Aut $G$ of $G$ for a group $G$. In particular, we concentrate on the case where $G$ is a free group and a free metabelian group. We also review the definition of the Magnus representations of $\mathrm{IA}_{n}$ and $\mathrm{IA}_{n}^{M}$. In Section 3. we determine the cokernel of the Johnson homomorphisms of the automorphism group of a free metabelian group. In Section 4, we show that the image of the cup product map $\cup_{\mathbf{Q}}^{M}$ is isomorphic to that of $\cup_{\mathbf{Q}}$. Finally, in Section 5 , we determine the $\mathrm{GL}(n, \mathbf{Z})$-module structure of $\overline{\mathcal{K}}_{n}^{\mathbf{Q}}$ and show that $\cup_{\mathbf{Q}}^{M}$ is not surjective.

\section{Preliminaries}

In this section, we recall the definition and some properties of the associated Lie algebra, the IA-automorphism group of $G$, and the Johnson homomorphisms of the automorphism group Aut $G$ of $G$ for any group $G$. In Subsections 2.2 and 2.3, we consider the case where $G$ is a free group and a free metabelian group.

2.1. Notation. First of all, throughout this paper we use the following notation and conventions.

- For a group $G$, the abelianization of $G$ is denoted by $G^{\mathrm{ab}}$.

- For a group $G$, the group Aut $G$ acts on $G$ from the right. For any $\sigma \in \operatorname{Aut} G$ and $x \in G$, the action of $\sigma$ on $x$ is denoted by $x^{\sigma}$.

- For a group $G$ and its quotient group $G / N$, we also denote the coset class of an element $g \in G$ by $g \in G / N$ if there is no confusion.

- For any $\mathbf{Z}$-module $M$, we denote $M \otimes_{\mathbf{Z}} \mathbf{Q}$ by the symbol obtained by attaching a subscript $\mathbf{Q}$ to $M$, such as $M_{\mathbf{Q}}$ or $M^{\mathbf{Q}}$. Similarly, for any $\mathbf{Z}$ linear map $f: A \rightarrow B$, the induced $\mathbf{Q}$-linear map $A_{\mathbf{Q}} \rightarrow B_{\mathbf{Q}}$ is denoted by $f_{\mathbf{Q}}$ or $f^{\mathbf{Q}}$.

- For elements $x$ and $y$ of a group, the commutator bracket $[x, y]$ of $x$ and $y$ is defined to be $[x, y]:=x y x^{-1} y^{-1}$. 
2.1.1. Associated Lie algebra of a group. For a group $G$, we define the lower central series of $G$ by the rule

$$
\Gamma_{G}(1):=F_{n}, \quad \Gamma_{G}(k):=\left[\Gamma_{G}(k-1), G\right], \quad k \geq 2 .
$$

We denote by $\mathcal{L}_{G}(k):=\Gamma_{G}(k) / \Gamma_{G}(k+1)$ the graded quotient of the lower central series of $G$, and by $\mathcal{L}_{G}:=\bigoplus_{k>1} \mathcal{L}_{G}(k)$ the associated graded sum. The graded sum $\mathcal{L}_{G}$ naturally has a graded Lie algebra structure induced from the commutator bracket on $G$, and is called the associated Lie algebra of $G$.

For any $g_{1}, \ldots, g_{t} \in G$, a commutator of weight $k$ type of

$$
\left[\left[\cdots\left[\left[g_{i_{1}}, g_{i_{2}}\right], g_{i_{3}}\right], \cdots\right], g_{i_{k}}\right], \quad i_{j} \in\{1, \ldots, t\},
$$

with all of its brackets to the left of all the elements occuring, is called a simple $k$-fold commutator among the components $g_{1}, \ldots, g_{t}$, and we denote it by

$$
\left[g_{i_{1}}, g_{i_{2}}, \ldots, g_{i_{k}}\right]
$$

for simplicity. Then we have

Lemma 2.1. If $G$ is generated by $g_{1}, \ldots, g_{t}$, then each of the graded quotients $\Gamma_{G}(k) / \Gamma_{G}(k+1)$ is generated by the simple $k$-fold commutators

$$
\left[g_{i_{1}}, g_{i_{2}}, \ldots, g_{i_{k}}\right], \quad i_{j} \in\{1, \ldots, t\} .
$$

Let $\rho_{G}:$ Aut $G \rightarrow$ Aut $G^{\text {ab }}$ be the natural homomorphism induced from the abelianization of $G$. The kernel $\operatorname{IA}(G)$ of $\rho_{G}$ is called the IA-automorphism group of $G$. Then the automorphism group Aut $G$ naturally acts on $\mathcal{L}_{G}(k)$ for each $k \geq 1$, and $\operatorname{IA}(G)$ acts on it trivially. Hence the action of Aut $G / \operatorname{IA}(G)$ on $\mathcal{L}_{G}(k)$ is welldefined.

2.1.2. Johnson homomorphisms. For $k \geq 0$, the action of Aut $G$ on each nilpotent quotient $G / \Gamma_{G}(k+1)$ induces a homomorphism

$$
\rho_{G}^{k}: \operatorname{Aut} G \rightarrow \operatorname{Aut}\left(G / \Gamma_{G}(k+1)\right) .
$$

The map $\rho_{G}^{0}$ is trivial, and $\rho_{G}^{1}=\rho_{G}$. We denote the kernel of $\rho_{G}^{k}$ by $\mathcal{A}_{G}(k)$. Then the groups $\mathcal{A}_{G}(k)$ define a descending central filtration

$$
\text { Aut } G=\mathcal{A}_{G}(0) \supset \mathcal{A}_{G}(1) \supset \mathcal{A}_{G}(2) \supset \cdots
$$

of Aut $G$, with $\mathcal{A}_{G}(1)=\operatorname{IA}(G)$. (See [1] for details.) We call it the Johnson filtration of Aut $G$. For each $k \geq 1$, the group Aut $G$ acts on $\mathcal{A}_{G}(k)$ by conjugation,

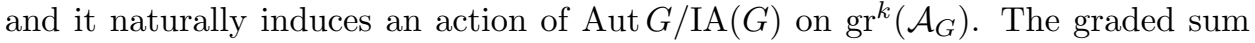
$\operatorname{gr}\left(\mathcal{A}_{G}\right):=\bigoplus_{k \geq 1} \operatorname{gr}^{k}\left(\mathcal{A}_{G}\right)$ has a graded Lie algebra structure induced from the commutator bracket on $\operatorname{IA}(G)$.

To study the Aut $G / \operatorname{IA}(G)$-module structure of each graded quotient $\operatorname{gr}^{k}\left(\mathcal{A}_{G}\right)$, we define the Johnson homomorphisms of Aut $G$ in the following way. For each $k \geq 1$, we consider a map $\mathcal{A}_{G}(k) \rightarrow \operatorname{Hom}_{\mathbf{Z}}\left(G^{\mathrm{ab}}, \mathcal{L}_{G}(k+1)\right)$ defined by

$$
\sigma \mapsto\left(g \mapsto g^{-1} g^{\sigma}\right), \quad x \in G .
$$

Then the kernel of this homomorphism is just $\mathcal{A}_{G}(k+1)$. Hence it induces an injective homomorphism

$$
\tau_{k}=\tau_{G, k}: \operatorname{gr}^{k}\left(\mathcal{A}_{G}\right) \hookrightarrow \operatorname{Hom}_{\mathbf{Z}}\left(G^{\mathrm{ab}}, \mathcal{L}_{G}(k+1)\right) .
$$

The homomorphsim $\tau_{k}$ is called the $k$-th Johnson homomorphism of Aut $G$. It is easily seen that each $\tau_{k}$ is an $\operatorname{Aut} G / \operatorname{IA}(G)$-equivariant homomorphism. Since 
each Johnson homomorphism $\tau_{k}$ is injective, to determine the cokernel of $\tau_{k}$ is an important problem for the study of the structure of $\operatorname{gr}^{k}\left(\mathcal{A}_{G}\right)$ as an $\operatorname{Aut} G / \operatorname{IA}(G)$ module.

Here, we consider another descending filtration of $\operatorname{IA}(G)$. Let $\mathcal{A}_{G}^{\prime}(k)$ be the $k$-th subgroup of the lower central series of $\operatorname{IA}(G)$. Then for each $k \geq 1, \mathcal{A}_{G}^{\prime}(k)$ is a subgroup of $\mathcal{A}_{G}(k)$ since $\mathcal{A}_{G}(k)$ is a central filtration of $\mathrm{IA}(G)$. In general, it is not known whether or not $\mathcal{A}_{G}^{\prime}(k)$ coincides with $\mathcal{A}_{G}(k)$. Set $\operatorname{gr}^{k}\left(\mathcal{A}_{G}^{\prime}\right):=$ $\mathcal{A}_{G}^{\prime}(k) / \mathcal{A}_{G}^{\prime}(k+1)$ for each $k \geq 1$. The restriction of the homomorphism $\mathcal{A}_{G}(k) \rightarrow$ $\operatorname{Hom}_{\mathbf{Z}}\left(G^{\mathrm{ab}}, \mathcal{L}_{G}(k+1)\right)$ to $\mathcal{A}_{G}^{\prime}(k)$ induces an $\operatorname{Aut} G / \mathrm{IA}(G)$-equivariant homomorphism

$$
\tau_{k}^{\prime}=\tau_{G, k}^{\prime}: \operatorname{gr}^{k}\left(\mathcal{A}_{G}^{\prime}\right) \rightarrow \operatorname{Hom}_{\mathbf{Z}}\left(G^{\mathrm{ab}}, \mathcal{L}_{G}(k+1)\right) .
$$

In this paper, we also call $\tau_{k}^{\prime}$ the $k$-th Johnson homomorphism of Aut $G$.

For any $\sigma \in \mathcal{A}_{G}(k)$ and $\tau \in \mathcal{A}_{G}(l)$, we give an example of computation of $\tau_{k+l}([\sigma, \tau])$ using $\tau_{k}(\sigma)$ and $\tau_{l}(\tau)$. For $\sigma \in \mathcal{A}_{G}(k)$ and $g \in G$, set $s_{g}(\sigma):=g^{-1} g^{\sigma} \in$ $\Gamma_{G}(k+1)$. Then, $\tau_{k}(\sigma)(g)=s_{g}(\sigma) \in \mathcal{L}_{G}(k+1)$. For any $\sigma \in \mathcal{A}_{G}(k)$ and $\tau \in \mathcal{A}_{G}(l)$, by an easy calculation we have

$$
\begin{aligned}
s_{g}([\sigma, \tau]) & =\left(s_{g}(\tau)^{-1}\right)^{\tau^{-1}}\left(s_{g}(\sigma)^{-1}\right)^{\sigma^{-1} \tau^{-1}} s_{g}(\tau)^{\sigma^{-1} \tau^{-1}} s_{g}(\sigma)^{\tau \sigma^{-1} \tau^{-1}} \\
& \equiv s_{g}(\sigma)^{-1} s_{g}(\sigma)^{\tau} \cdot\left(s_{g}(\tau)^{-1} s_{g}(\tau)^{\sigma}\right)^{-1} \quad\left(\bmod \Gamma_{G}(k+l+2)\right) .
\end{aligned}
$$

Using this fomula, we can easily compute $s_{g}([\sigma, \tau])$ from $s_{g}(\sigma)$ and $s_{g}(\tau)$. For example, if $s_{g}(\sigma)$ and $s_{g}(\tau)$ is given by

(2) $s_{g}(\sigma)=\left[g_{1}, g_{2}, \ldots, g_{k+1}\right] \in \mathcal{L}_{G}(k+1), \quad s_{g}(\tau)=\left[h_{1}, h_{2}, \ldots, h_{l+1}\right] \in \mathcal{L}_{G}(l+1)$,

then we obtain

$$
s_{g}([\sigma, \tau])=\left(\sum_{i=1}^{k+1}\left[g_{1}, \ldots, s_{g_{i}}(\tau), \ldots, g_{k+1}\right]\right)-\left(\sum_{j=1}^{l+1}\left[h_{1}, \ldots, s_{h_{j}}(\sigma), \ldots, h_{l+1}\right]\right)
$$

in $\mathcal{L}_{G}(k+l+1)$.

2.2. Free groups. In this section we consider the case where $G$ is a free group of finite rank.

2.2.1. Free Lie algebra. For $n \geq 2$, let $F_{n}$ be a free group of rank $n$ with basis $x_{1}, \ldots, x_{n}$. We denote the abelianization of $F_{n}$ by $H$ and its dual group by $H^{*}:=$ $\operatorname{Hom}_{\mathbf{Z}}(H, \mathbf{Z})$. If we fix the basis of $H$ as a free abelian group induced from the basis $x_{1}, \ldots, x_{n}$ of $F_{n}$, we can identify $\operatorname{Aut} F_{n}^{\mathrm{ab}}=\operatorname{Aut}(H)$ with the general linear group $\mathrm{GL}(n, \mathbf{Z})$. Furthermore, it is classically well known that the map $\rho_{F_{n}}$ : Aut $F_{n} \rightarrow \mathrm{GL}(n, \mathbf{Z})$ is surjective. (See [21, proposition 4.4.) Hence we also identify $\operatorname{Aut}(H) / \operatorname{IA}\left(F_{n}\right)$ with $\operatorname{GL}(n, \mathbf{Z})$. In this paper, for simplicity, we write $\Gamma_{n}(k), \mathcal{L}_{n}(k)$ and $\mathcal{L}_{n}$ for $\Gamma_{F_{n}}(k), \mathcal{L}_{F_{n}}(k)$ and $\mathcal{L}_{F_{n}}$ respectively.

The associated Lie algebra $\mathcal{L}_{n}$ is called the free Lie algebra generated by $H$. (See 32 for basic material concerning free Lie algebra.) It is classically well known due to Witt 34 that each $\mathcal{L}_{n}(k)$ is a $\mathrm{GL}(n, \mathbf{Z})$-equivariant free abelian group of rank

$$
r_{n}(k):=\frac{1}{k} \sum_{d \mid k} \mu(d) n^{\frac{k}{d}}
$$

where $\mu$ is the Möbius function. 
Next we consider the $\operatorname{GL}(n, \mathbf{Z})$-module structure of $\mathcal{L}_{n}(k)$. For example, for $1 \leq k \leq 3$ we have

$$
\begin{aligned}
& \mathcal{L}_{n}(1)=H, \quad \mathcal{L}_{n}(2)=\Lambda^{2} H \\
& \mathcal{L}_{n}(3)=\left(H \otimes \mathbf{z} \Lambda^{2} H\right) /\langle x \otimes y \wedge z+y \otimes z \wedge x+z \otimes x \wedge y \mid x, y, z \in H\rangle .
\end{aligned}
$$

In general, the irreducible decomposition of $\mathcal{L}_{n}^{\mathbf{Q}}(k)$ as a $\mathrm{GL}(n, \mathbf{Z})$-module is completely determined. For $k \geq 1$ and any Young diagram $\lambda=\left[\lambda_{1}, \ldots, \lambda_{l}\right]$ of degree $k$, let $H^{\lambda}$ be the Schur-Weyl module of $H$ corresponding to the Young diagram $\lambda$. For example, $H^{[k]}=S^{k} H$ and $H^{\left[1^{k}\right]}=\Lambda^{k} H$. (For details, see [12] and [13.) Let $m\left(H_{\mathbf{Q}}^{\lambda}, \mathcal{L}_{n}^{\mathbf{Q}}(k)\right)$ be the multiplicity of the Schur-Weyl module $H_{\mathbf{Q}}^{\lambda}$ in $\mathcal{L}_{n}^{\mathbf{Q}}(k)$. Bakhturin [6] gave a formula for $m\left(H_{\mathbf{Q}}^{\lambda}, \mathcal{L}_{n}^{\mathbf{Q}}(k)\right)$ using the character of the Specht module of $H_{\mathbf{Q}}$ corresponding to the Young diagram $\lambda$. However, its character value had remained unknown in general. Then Zhuravlev 35] gave a method of calculation for it. Using these facts, we can give the explicit irreducible decomposition of $\mathcal{L}_{n}^{\mathbf{Q}}(k)$. For example,

$$
\mathcal{L}_{n}^{\mathbf{Q}}(3) \cong H_{\mathbf{Q}}^{[2,1]}, \quad \mathcal{L}_{n}^{\mathbf{Q}}(4) \cong H_{\mathbf{Q}}^{[3,1]} \oplus H_{\mathbf{Q}}^{[2,1,1]} .
$$

2.2.2. IA-automorphism group of a free group. Now we consider the IA-automorphism group of $F_{n}$. We denote $\operatorname{IA}\left(F_{n}\right)$ by $\mathrm{IA}_{n}$. It is well known due to Nielsen 27. that $\mathrm{IA}_{2}$ coincides with the inner automorphsim group $\operatorname{Inn} F_{2}$ of $F_{2}$. Namely, $\mathrm{IA}_{2}$ is a free group of rank 2 . However, $\mathrm{IA}_{n}$ for $n \geq 3$ is much larger than $\operatorname{Inn} F_{n}$. Indeed, Magnus [22] showed that for any $n \geq 3$, the IA-automorphism group $\mathrm{IA}_{n}$ is finitely generated by automorphisms

$$
K_{i j}:\left\{\begin{array}{ll}
x_{i} & \mapsto x_{j}{ }^{-1} x_{i} x_{j}, \\
x_{t} & \mapsto x_{t}
\end{array} \quad(t \neq i)\right.
$$

for distinct $i, j \in\{1,2, \ldots, n\}$ and

$$
K_{i j k}:\left\{\begin{array}{lll}
x_{i} & \mapsto x_{i} x_{j} x_{k} x_{j}{ }^{-1} x_{k}{ }^{-1}, \\
x_{t} & \mapsto x_{t} \quad(t \neq i)
\end{array}\right.
$$

for distinct $i, j, k \in\{1,2, \ldots, n\}$ such that $j<k$.

For any $n \geq 3$, although a generating set of $\mathrm{IA}_{n}$ is well known as above, any presentation for $\mathrm{IA}_{n}$ is still not known. For $n=3$, Krstić and McCool 20, showed that $\mathrm{IA}_{3}$ is not finitely presentable. For $n \geq 4$, it is also not known whether or not $\mathrm{IA}_{n}$ is finitely presentable.

Andreadakis [1] showed that the first Johnson homomorphism $\tau_{1}$ of Aut $F_{n}$ is surjective by computing the image of the generators of $\mathrm{IA}_{n}$ above. Furthermore, recently, Cohen-Pakianathan [9, 10, Farb [11] and Kawazumi [19] independently showed that $\tau_{1}$ induces the abelianization of $\mathrm{IA}_{n}$. Namely, for any $n \geq 3$, we have

$$
\mathrm{IA}_{n}^{\mathrm{ab}} \cong H^{*} \otimes \mathbf{z} \Lambda^{2} H
$$

as a $\operatorname{GL}(n, \mathbf{Z})$-module.

2.2.3. Johnson homomorphisms of Aut $F_{n}$. Here, we consider the Johnson homomorphisms of Aut $F_{n}$. Throughout this paper, for simplicity, we write $\mathcal{A}_{n}(k)$, 
$\mathcal{A}_{n}^{\prime}(k), \operatorname{gr}^{k}\left(\mathcal{A}_{n}\right)$ and $\operatorname{gr}^{k}\left(\mathcal{A}_{n}^{\prime}\right)$ for $\mathcal{A}_{F_{n}}(k), \mathcal{A}_{F_{n}}^{\prime}(k), \operatorname{gr}^{k}\left(\mathcal{A}_{F_{n}}\right)$ and $\operatorname{gr}^{k}\left(\mathcal{A}_{F_{n}}^{\prime}\right)$ respectively. Pettet [31] showed

$$
\operatorname{rank}_{\mathbf{Z}} \operatorname{gr}^{2}\left(\mathcal{A}_{n}\right)=\frac{1}{6} n(n+1)\left(2 n^{2}-2 n-3\right),
$$

and in our previous paper 33, we showed

$$
\operatorname{rank}_{\mathbf{Z}} \operatorname{gr}^{3}\left(\mathcal{A}_{n}\right)=\frac{1}{12} n\left(3 n^{4}-7 n^{2}-8\right) .
$$

In general, for any $n \geq 3$ and $k \geq 4$ the rank of $\operatorname{gr}^{k}\left(\mathcal{A}_{n}\right)$ is still not known. One of the aims of this paper is to give a lower bound on $\operatorname{rank}_{\mathbf{Z}} \operatorname{gr}^{k}\left(\mathcal{A}_{n}\right)$ by studying the Johnson filtration of the automorphism group of a free metabelian group.

Next, we mention the relation between $\mathcal{A}_{n}^{\prime}(k)$ and $\mathcal{A}_{n}(k)$. Since $\tau_{1}$ is the abelianization of $\mathrm{IA}_{n}$ as mentioned above, we have $\mathcal{A}_{n}^{\prime}(2)=\mathcal{A}_{n}(2)$. Furthermore, Pettet 31] showed that $\mathcal{A}_{n}^{\prime}(3)$ has at most finite index in $\mathcal{A}_{n}(3)$. Although it is conjectured that $\mathcal{A}_{n}^{\prime}(k)=\mathcal{A}_{n}(k)$ for $k \geq 3$, there are few results for the difference between $\mathcal{A}_{n}^{\prime}(k)$ and $\mathcal{A}_{n}(k)$ for $n \geq 3$.

Let $H^{*}$ be the dual group $\operatorname{Hom}_{\mathbf{Z}}(H, \mathbf{Z})$ of $H$. For the standard basis $x_{1}, \ldots, x_{n}$ of $H$ induced from the generators of $F_{n}$, let $x_{1}^{*}, \ldots, x_{n}^{*}$ be its dual basis of $H^{*}$. Then identifying $\operatorname{Hom}_{\mathbf{Z}}\left(H, \mathcal{L}_{n}(k+1)\right)$ with $H^{*} \otimes_{\mathbf{Z}} \mathcal{L}_{n}(k+1)$, we obtain the Johnson homomorphism

$$
\tau_{k}: \operatorname{gr}^{k}\left(\mathcal{A}_{n}\right) \hookrightarrow H^{*} \otimes \mathbf{z} \mathcal{L}_{n}(k+1)
$$

of Aut $F_{n}$. Here we give some examples of computation $\tau_{k}(\sigma)$ for $\sigma \in \mathcal{A}_{n}(k)$. For the generators $K_{i j}$ and $K_{i j k}$ of $\mathcal{A}_{n}(1)=\mathrm{IA}_{n}$, we have

$$
s_{x_{l}}\left(K_{i j}\right)=\left\{\begin{array}{ll}
1, & l \neq i, \\
{\left[x_{i}^{-1}, x_{j}^{-1}\right],} & l=i,
\end{array} \quad s_{x_{l}}\left(K_{i j k}\right)= \begin{cases}1, & l \neq i, \\
{\left[x_{j}, x_{k}\right],} & l=i\end{cases}\right.
$$

in $\Gamma_{n}(2)$. Hence

$$
\tau_{1}\left(K_{i j}\right)=x_{i}^{*} \otimes\left[x_{i}, x_{j}\right], \quad \tau_{1}\left(K_{i j k}\right)=x_{i}^{*} \otimes\left[x_{j}, x_{k}\right]
$$

in $H^{*} \otimes \mathbf{z} \mathcal{L}_{n}(2)$. Then using (1) and (7), we can recursively compute $\tau_{k}(\sigma)=\tau_{k}^{\prime}(\sigma)$ for $\sigma \in \mathcal{A}_{n}^{\prime}(k)$. These computations are perhaps easiest explained with examples, so we give two here. For distinct $a, b, c$ and $d$ in $\{1,2, \ldots, n\}$, we have

$$
\begin{aligned}
& \tau_{2}^{\prime}\left(\left[K_{a b}, K_{b a c}\right]\right)=x_{a}^{*} \otimes( {\left.\left[s_{x_{a}}\left(K_{b a c}\right), x_{b}\right]+\left[x_{a}, s_{x_{b}}\left(K_{b a c}\right)\right]\right) } \\
&-x_{b}^{*} \otimes\left(\left[s_{x_{a}}\left(K_{a b}\right), x_{c}\right]+\left[x_{a}, s_{x_{c}}\left(K_{a b}\right)\right]\right) \\
&=x_{a}^{*} \otimes\left[x_{a},\left[x_{a}, x_{c}\right]\right]-x_{b}^{*} \otimes\left[\left[x_{a}, x_{b}\right], x_{c}\right]
\end{aligned}
$$

and

$$
\begin{aligned}
\tau_{3}^{\prime}\left(\left[K_{a b}, K_{b a c},\right.\right. & \left.\left.K_{a d}\right]\right) \\
=x_{a}^{*} & \otimes\left(\left[s_{x_{a}}\left(K_{a d}\right),\left[x_{a}, x_{c}\right]\right]+\left[x_{a},\left[s_{x_{a}}\left(K_{a d}\right), x_{c}\right]\right]+\left[x_{a},\left[x_{a}, s_{x_{c}}\left(K_{a d}\right)\right]\right]\right) \\
& \quad-x_{b}^{*} \otimes\left(\left[\left[s_{x_{a}}\left(K_{a d}\right), x_{b}\right], x_{c}\right]+\left[\left[x_{a}, s_{x_{b}}\left(K_{a d}\right)\right], x_{c}\right]+\left[\left[x_{a}, x_{b}\right], s_{x_{c}}\left(K_{a d}\right)\right]\right) \\
& \quad-x_{a}^{*} \otimes\left(\left[s_{x_{a}}\left(\left[K_{a b}, K_{b a c}\right]\right), x_{d}\right]+\left[x_{a}, s_{x_{d}}\left(\left[K_{a b}, K_{b a c}\right]\right)\right]\right) \\
= & x_{a}^{*} \otimes\left[\left[x_{a}, x_{d}\right],\left[x_{a}, x_{c}\right]\right]+x_{a}^{*} \otimes\left[x_{a},\left[\left[x_{a}, x_{d}\right], x_{c}\right]\right] \\
& -x_{b}^{*} \otimes\left[\left[\left[x_{a}, x_{d}\right], x_{b}\right], x_{c}\right] \\
& -x_{a}^{*} \otimes\left[\left[x_{a},\left[x_{a}, x_{c}\right]\right], x_{d}\right] .
\end{aligned}
$$

2.3. Free metabelian groups. In this section we consider the case where a group $G$ is a free metabelian group of finite rank. 
2.3.1. Free metabelian Lie algebra. Let $F_{n}^{M}=F_{n} / F_{n}^{\prime \prime}$ be a free metabelian group of rank $n$ where $F_{n}^{\prime \prime}=\left[\left[F_{n}, F_{n}\right],\left[F_{n}, F_{n}\right]\right]$ is the second derived group of $F_{n}$. Then we have $\left(F_{n}^{M}\right)^{\mathrm{ab}}=H$, and hence Aut $\left(F_{n}^{M}\right)^{\mathrm{ab}}=\operatorname{Aut}(H)=\operatorname{GL}(n, \mathbf{Z})$. Since the surjective map $\rho_{F_{n}}:$ Aut $F_{n} \rightarrow \operatorname{GL}(n, \mathbf{Z})$ factors through Aut $F_{n}^{M}$, a map $\rho_{F_{n}^{M}}$ : Aut $F_{n}^{M} \rightarrow \mathrm{GL}(n, \mathbf{Z})$ is also surjective. Hence we identify Aut $F_{n}^{M} / \operatorname{IA}\left(F_{n}^{M}\right)$ with $\mathrm{GL}(n, \mathbf{Z})$. In this paper, for simplicity, we write $\Gamma_{n}^{M}(k), \mathcal{L}_{n}^{M}(k)$ and $\mathcal{L}_{n}^{M}$ for $\Gamma_{F_{n}^{M}}(k)$, $\mathcal{L}_{F_{n}^{M}}(k)$ and $\mathcal{L}_{F_{n}^{M}}$ respectively.

The associated Lie algebra $\mathcal{L}_{n}^{M}$ is called the free metabelian algebra generated by $H$. We see that $\mathcal{L}_{n}(k)=\mathcal{L}_{n}^{M}(k)$ for $1 \leq k \leq 3$. It is also classically well known due to Chen 8 that each $\mathcal{L}_{n}^{M}(k)$ is a $\operatorname{GL}(n, \mathbf{Z})$-equivariant free abelian group of rank

$$
r_{n}^{M}(k):=(k-1)\left(\begin{array}{c}
n+k-2 \\
k
\end{array}\right)
$$

2.3.2. IA-automorphism group of a free metabelian group. Here we consider the IAautomorphism group of $F_{M}$. Let $\mathrm{IA}_{n}^{M}:=\mathrm{IA}\left(F_{n}^{M}\right)$. We denote by $\nu_{n}:$ Aut $F_{n} \rightarrow$ Aut $F_{n}^{M}$ the natural homomorphism induced from the action of Aut $F_{n}$ on $F_{n}^{M}$. Restricting $\nu_{n}$ to $\mathrm{IA}_{n}$, we obtain a homomorphism $\nu_{n, 1}: \mathrm{IA}_{n} \rightarrow \mathrm{IA}_{n}^{M}$. Bachmuth and Mochizuki 44 showed that $\nu_{3,1}$ is not surjective and $\mathrm{IA}_{3}^{M}$ is not finitely generated. They also showed that in [5], $\nu_{n, 1}$ is surjective for $n \geq 4$. Hence $\mathrm{IA}_{n}^{M}$ is finitely generated for $n \geq 4$. It is, however, not known whether or not $\mathrm{IA}_{n}^{M}$ is finitely presented for $n \geq 4$.

From now on, we consider the case where $n \geq 4$. Set $\mathcal{K}_{n}:=\operatorname{Ker}\left(\nu_{n}\right)$. Since $\mathcal{K}_{n} \subset \mathrm{IA}_{n}$, we have an exact sequence

$$
1 \rightarrow \mathcal{K}_{n} \rightarrow \mathrm{IA}_{n} \rightarrow \mathrm{IA}_{n}^{M} \rightarrow 1 .
$$

Furthermore, observing $\mathcal{K}_{n} \subset \mathcal{A}_{n}(2)=\left[\mathrm{IA}_{n}, \mathrm{IA}_{n}\right]$, we obtain

$$
\left(\mathrm{IA}_{n}^{M}\right)^{\mathrm{ab}} \cong \mathrm{IA}_{n}^{\mathrm{ab}} \cong H^{*} \otimes_{\mathbf{Z}} \Lambda^{2} H
$$

and see that the first Johnson homomorphism $\tau_{1}$ of Aut $F_{n}^{M}$ is an isomorphism.

2.3.3. Johnson homomorphisms of Aut $F_{n}^{M}$. Here we consider the Johnson homomorphisms of Aut $\left(F_{n}^{M}\right)$. We denote $\mathcal{A}_{F_{n}^{M}}(k)$ and $\operatorname{gr}^{k}\left(\mathcal{A}_{F_{n}^{M}}\right)$ by $\mathcal{A}_{n}^{M}(k)$ and $\operatorname{gr}^{k}\left(\mathcal{A}_{n}^{M}\right)$ respectively. Furthermore, we also denote $\mathcal{A}_{F_{n}^{M}}^{\prime}(k)$ and $\operatorname{gr}^{k}\left(\mathcal{A}_{F_{n}^{M}}^{\prime}\right)$ by $\mathcal{A}_{n}^{\prime M}(k)$ and $\operatorname{gr}^{k}\left(\mathcal{A}_{n}^{\prime M}\right)$ respectively.

For each $k \geq 1$, restricting $\nu_{n}$ to $\mathcal{A}_{n}(k)$, we obtain a homomorphism $\nu_{n, k}$ : $\mathcal{A}_{n}(k) \rightarrow \mathcal{A}_{n}^{M}(k)$. Since $\tau_{1}: \operatorname{gr}^{1}\left(\mathcal{A}_{n}^{\prime} M\right) \rightarrow H^{*} \otimes_{\mathbf{z}} \Lambda^{2} H$ is an isomorphism, we see that $\mathcal{A}_{n}^{M}(2)=\mathcal{A}_{n}^{\prime M}(2)$, and hence $\nu_{n, 2}$ is surjective. However it is not known whether or not $\nu_{n, k}$ is surjective for $k \geq 3$.

Now, the main aim of the paper is to determine the $\operatorname{GL}(n, \mathbf{Z})$-module structure of the cokernel of the Johnson homomorphisms of Aut $F_{n}^{M}$. In this paper, we give an answer to this problem for the case where $k \geq 2$ and $n \geq 4$. We remark that by an argument similar to that in Subsection 2.2, we can recursively compute $\tau_{k}(\sigma)=$ $\tau_{k}^{\prime}(\sigma)$ for $\sigma \in \mathcal{A}_{n}^{\prime M}(k)$, using $\tau_{1}\left(\nu_{n, 1}\left(K_{i j}\right)\right)=x_{i}^{*} \otimes\left[x_{i}, x_{j}\right]$ and $\tau_{1}\left(\nu_{n, 1}\left(K_{i j k}\right)\right)=$ $x_{i}^{*} \otimes\left[x_{j}, x_{k}\right]$. 
2.4. Magnus representations. In this subsection we recall the Magnus representation of Aut $F_{n}$ and Aut $F_{n}^{M}$. (For details, see [7.) For each $1 \leq i \leq n$, let

$$
\frac{\partial}{\partial x_{i}}: \mathbf{Z}\left[F_{n}\right] \rightarrow \mathbf{Z}\left[F_{n}\right]
$$

be the Fox derivation defined by

$$
\frac{\partial}{\partial x_{i}}(w)=\sum_{j=1}^{r} \epsilon_{j} \delta_{\mu_{j}, i} x_{\mu_{1}}^{\epsilon_{1}} \cdots x_{\mu_{j}}^{\frac{1}{2}\left(\epsilon_{j}-1\right)} \in \mathbf{Z}\left[F_{n}\right]
$$

for any reduced word $w=x_{\mu_{1}}^{\epsilon_{1}} \cdots x_{\mu_{r}}^{\epsilon_{r}} \in F_{n}, \epsilon_{j}= \pm 1$. Let $\mathfrak{a}: F_{n} \rightarrow H$ be the abelianization of $F_{n}$. We also denote by $\mathfrak{a}$ the ring homomorphism $\mathbf{Z}\left[F_{n}\right] \rightarrow \mathbf{Z}[H]$ induced from $\mathfrak{a}$. For any $A=\left(a_{i j}\right) \in \mathrm{GL}\left(n, \mathbf{Z}\left[F_{n}\right]\right)$, let $A^{\mathfrak{a}}$ be the matrix $\left(a_{i j}^{\mathfrak{a}}\right) \in$ $\mathrm{GL}(n, \mathbf{Z}[H])$. The Magnus representation $\overline{\operatorname{rep}}: \operatorname{Aut} F_{n} \rightarrow \mathrm{GL}(n, \mathbf{Z}[H])$ of Aut $F_{n}$ is defined by

$$
\sigma \mapsto\left(\frac{\partial x_{i} \sigma}{\partial x_{j}}\right)^{\mathfrak{a}}
$$

for any $\sigma \in \operatorname{Aut} F_{n}$. This map is not a homomorphism but a crossed homomorphism. Namely,

$$
\overline{\operatorname{rep}}(\sigma \tau)=(\overline{\operatorname{rep}}(\sigma))^{\tau^{*}} \cdot \overline{\operatorname{rep}}(\tau),
$$

where $(\overline{\mathrm{rep}}(\sigma))^{*}$ denotes the matrix obtained from $\overline{\operatorname{rep}}(\sigma)$ by applying the automorphism $\tau^{*}: \mathbf{Z}[H] \rightarrow \mathbf{Z}[H]$ induced from $\rho(\tau) \in \operatorname{Aut}(H)$ on each entry. Hence by restricting $\overline{\text { rep }}$ to $\mathrm{IA}_{n}$, we obtain a homomorphism rep : $\mathrm{IA}_{n} \rightarrow \mathrm{GL}(n, \mathbf{Z}[H])$. This is called the Magnus representation of $\mathrm{IA}_{n}$.

Next, we consider the Magnus representation of $\mathrm{IA}_{n}^{M}$. Let rep ${ }^{M}: \mathrm{IA}_{n}^{M} \rightarrow$ $\mathrm{GL}(n, \mathbf{Z}[H])$ be a map defined by

$$
\sigma \mapsto\left(\frac{\partial\left(x_{i}{ }^{\sigma}\right)}{\partial x_{j}}\right)^{\mathfrak{a}}
$$

for any $\sigma \in \mathrm{IA}_{n}^{M}$, where we consider any lift of the element $x_{i}{ }^{\sigma} \in F_{n}^{M}$ to $F_{n}$. Then we see $\operatorname{rep}^{M}$ is a homomorphism and rep $=\operatorname{rep}^{M} \circ \nu_{n, 1}$, and call it the Magnus representation of $\mathrm{IA}_{n}^{M}$. Bachmuth [2] showed that rep $^{M}$ is faithful, and determined the image of $\operatorname{rep}^{M}$ in $\operatorname{GL}(n, \mathbf{Z}[H])$. The faithfulness of the Magnus representation rep $^{M}$ shows that the kernel of the Magnus representation rep is equal to $\mathcal{K}_{n}$.

\section{The COKERNEL OF THE JOHNSON HOMOMORPHISMS}

In this section, we determine the cokernel of the Johnson homomorphism $\tau_{k}$ of Aut $F_{n}^{M}$ for $k \geq 2$ and $n \geq 4$.

3.1. Upper bound on the rank of cokernel of $\tau_{k}$. First we give an upper bound on the rank of the cokernel of $\tau_{k}$ by reducing its set of generators. By Lemma 2.1. we see that elements of type $x_{i}^{*} \otimes\left[x_{i_{1}}, x_{i_{2}}, \ldots, x_{i_{k+1}}\right]$ generate $H^{*} \otimes \mathbf{z} \mathcal{L}_{n}^{M}(k+1)$. First we prepare some lemmas. Let $\mathfrak{S}_{l}$ be the symmetric group of degree $l$. Then we have

Lemma 3.1. Let $l \geq 2$ and $n \geq 2$. For any element $\left[x_{i_{1}}, x_{i_{2}}, x_{j_{1}}, \ldots, x_{j_{l}}\right] \in$ $\mathcal{L}_{n}^{M}(l+2)$ and any $\lambda \in \mathfrak{S}_{l}$,

$$
\left[x_{i_{1}}, x_{i_{2}}, x_{j_{1}}, \ldots, x_{j_{l}}\right]=\left[x_{i_{1}}, x_{i_{2}}, x_{j_{\lambda(1)}}, \ldots, x_{j_{\lambda(l)}}\right] .
$$


Proof. Since $\mathfrak{S}_{l}$ is generated by transpositions $(m m+1)$ for $1 \leq m \leq l-1$, it suffices to prove the lemma for each $\lambda=(m m+1)$. Now we have

$$
\begin{aligned}
{\left[\left[\left[x_{i_{1}}, x_{i_{2}}, x_{j_{1}}, \ldots, x_{j_{m-1}}\right], x_{j_{m}}\right], x_{j_{m+1}}\right] } & \\
= & -\left[\left[x_{j_{m}}, x_{j_{m+1}}\right],\left[x_{i_{1}}, x_{i_{2}}, x_{j_{1}}, \ldots, x_{j_{m-1}}\right]\right] \\
\quad & -\left[\left[x_{j_{m+1}},\left[x_{i_{1}}, x_{i_{2}}, x_{j_{1}}, \ldots, x_{j_{m-1}}\right]\right], x_{j_{m}}\right] \\
& =\left[\left[\left[x_{i_{1}}, x_{i_{2}}, x_{j_{1}}, \ldots, x_{j_{m-1}}\right], x_{j_{m+1}}\right], x_{j_{m}}\right]
\end{aligned}
$$

in $\mathcal{L}_{n}^{M}(m+3)$ by Jacobi's identity. Hence,

$$
\begin{aligned}
{\left[x_{i_{1}}, x_{i_{2}}, x_{j_{1}}, \ldots, x_{j_{l}}\right] } & =\left[x_{i_{1}}, x_{i_{2}}, x_{j_{1}}, \ldots, x_{j_{m-1}}, x_{j_{m+1}}, x_{j_{m}}, \ldots, x_{j_{l}}\right] \\
& =\left[x_{i_{1}}, x_{i_{2}}, x_{j_{\lambda(1)}} \ldots, x_{j_{\lambda(l)}}\right]
\end{aligned}
$$

in $\mathcal{L}_{n}^{M}(l+2)$.

Lemma 3.2. Let $k \geq 1$ and $n \geq 4$. For any $i$ and $i_{1}, i_{2}, \ldots, i_{k+1} \in\{1,2 \ldots, n\}$, if $i_{1}, i_{2} \neq i$,

$$
x_{i}^{*} \otimes\left[x_{i_{1}}, x_{i_{2}}, \ldots, x_{i_{k+1}}\right] \in \operatorname{Im}\left(\tau_{k}^{\prime}\right) .
$$

Proof. First, using Lemma 3.1. we remark that $\left[x_{i_{1}}, x_{i_{2}}, \ldots, x_{i_{k+1}}\right] \in \mathcal{L}_{n}^{M}(k+1)$ is rewritten as

$$
\left[x_{i_{1}}, x_{i_{2}}, x_{i_{3}}, \ldots, x_{i_{l-1}}, x_{i}, x_{i}, \ldots, x_{i}\right]
$$

in $\mathcal{L}_{n}^{M}(k+1)$ for some $l, 3 \leq l \leq k+2$. Namely, $i_{1}, i_{2}, \ldots, i_{l-1} \neq i$ and $i_{l}=i_{l+1}=$ $\cdots=i_{k+1}=i$.

We prove this lemma by induction on $k$. If $k=1$, by (7), we have $\tau_{1}^{\prime}\left(\nu_{n, 1}\left(K_{i i_{1} i_{2}}\right)\right)$ $=x_{i}^{*} \otimes\left[x_{i_{1}}, x_{i_{2}}\right]$. Suppose $k \geq 2$. Since $n \geq 4$, we can choose an element $j \in$ $\{1,2, \ldots, n\}$ such that $j \neq i, i_{1}, i_{2}$. Define $\sigma \in \mathcal{A}_{n}^{\prime M}(1)$ to be

$$
\sigma= \begin{cases}\nu_{n, 1}\left(K_{i j}^{-1}\right), & l \leq k+1 \\ \nu_{n, 1}\left(K_{i i_{k+1}}\right), & l=k+2 .\end{cases}
$$

Then

$$
\tau_{1}^{\prime}(\sigma)= \begin{cases}x_{i}^{*} \otimes\left[x_{j}, x_{i}\right], & l \leq k+1, \\ x_{i}^{*} \otimes\left[x_{i}, x_{i_{k+1}}\right], & l=k+2 .\end{cases}
$$

By the inductive hypothesis, we have an element $\tau \in \mathcal{A}_{n}^{\prime M}(k-1)$ such that

$$
\tau_{k-1}^{\prime}(\tau)= \begin{cases}x_{j}^{*} \otimes\left[x_{i_{1}}, x_{i_{2}}, x_{i_{3}}, \ldots, x_{i_{l-1}}, x_{i}, x_{i}, \ldots, x_{i}\right], & l \leq k+1, \\ x_{i}^{*} \otimes\left[x_{i_{1}}, x_{i_{2}}, x_{i_{3}}, \ldots, x_{i_{k+1}}\right], & l=k+2\end{cases}
$$

where $x_{i}$ appears $k-l+1$ times among the components of the commutator above. Then we obtain

$$
\tau_{k}^{\prime}([\sigma, \tau])=x_{i}^{*} \otimes\left[x_{i_{1}}, x_{i_{2}}, x_{i_{3}}, \ldots, x_{i_{l-1}}, x_{i}, x_{i}, \ldots, x_{i}\right],
$$

where $x_{i}$ appears $k-l+2$ times among the components. This completes the proof of Lemma 3.2

Lemma 3.3. Let $k \geq 1$ and $n \geq 4$. For any $i$ and $i_{1}, i_{2}, \ldots, i_{k} \in\{1,2, \ldots, n\}$ such that $i_{1}, i_{2} \neq i$, and any transposition $\lambda=(m m+1) \in \mathfrak{S}_{k}$,

$$
x_{i}^{*} \otimes\left[x_{i}, x_{i_{1}}, \ldots, x_{i_{k}}\right]-x_{i}^{*} \otimes\left[x_{i}, x_{i_{\lambda(1)}}, \ldots, x_{i_{\lambda(k)}}\right] \in \operatorname{Im}\left(\tau_{k}^{\prime}\right) .
$$


Proof. From Lemma 3.1, we have

$$
x_{i}^{*} \otimes\left[x_{i}, x_{i_{1}}, \ldots, x_{i_{k}}\right]-x_{i}^{*} \otimes\left[x_{i}, x_{i_{\lambda(1)}}, \ldots, x_{i_{\lambda(k)}}\right]=0
$$

in the case where $\lambda=(m m+1)$ for $2 \leq m \leq k-1$. Set $\lambda=(12)$. Since

$$
\left[\left[x_{i}, x_{i_{1}}\right], x_{i_{2}}\right]-\left[\left[x_{i}, x_{i_{2}}\right], x_{i_{1}}\right]=-\left[\left[x_{i_{1}}, x_{i_{2}}\right], x_{i}\right] \text {, }
$$

by the Jacobi's identity we have

$x_{i}^{*} \otimes\left[x_{i}, x_{i_{1}}, x_{i_{2}}, \ldots, x_{i_{k}}\right]-x_{i}^{*} \otimes\left[x_{i}, x_{i_{2}}, x_{i_{1}}, \ldots, x_{i_{k}}\right]=-x_{i}^{*} \otimes\left[x_{i_{1}}, x_{i_{2}}, x_{i}, \ldots, x_{i_{k}}\right]$.

Therefore Lemma 3.3 follows from Lemma 3.2 immediately.

Lemma 3.4. Let $k \geq 1$ and $n \geq 4$. For any $i_{2}, \ldots, i_{k+1} \in\{1,2, \ldots, n\}$, we have

$$
x_{i}^{*} \otimes\left[x_{i}, x_{i_{2}}, \ldots, x_{i_{k+1}}\right]-x_{j}^{*} \otimes\left[x_{j}, x_{i_{2}}, \ldots, x_{i_{k+1}}\right] \in \operatorname{Im}\left(\tau_{k}^{\prime}\right)
$$

for any $i \neq i_{2}$ and $j \neq i_{2}, i_{k+1}$.

Proof. We may assume $j \neq i$. First, set

$$
\tau= \begin{cases}\nu_{n, 1}\left(K_{i j i_{k+1}}\right), & \text { if } i_{k+1} \neq i, \\ \nu_{n, 1}\left(K_{i j}^{-1}\right), & \text { if } i_{k+1}=i .\end{cases}
$$

Then we have $\tau_{1}^{\prime}(\tau)=x_{i}^{*} \otimes\left[x_{j}, x_{i_{k+1}}\right]$. From Lemma 3.2, there exists a $\sigma \in \mathcal{A}_{n}^{\prime M}(k-1)$ such that

Hence we obtain

$$
\tau_{k-1}^{\prime}(\sigma)=x_{j}^{*} \otimes\left[x_{i}, x_{i_{2}}, \ldots, x_{i_{k}}\right] .
$$

$$
\tau_{k}^{\prime}([\tau, \sigma])=x_{i}^{*} \otimes\left[x_{i}, x_{i_{2}}, \ldots, x_{i_{k+1}}\right]-x_{j}^{*} \otimes\left[x_{j}, x_{i_{k+1}}, x_{i_{2}}, \ldots, x_{i_{k}}\right] .
$$

On the other hand, by Lemma 3.3, we have

$$
x_{j}^{*} \otimes\left[x_{j}, x_{i_{k+1}}, x_{i_{2}}, \ldots, x_{i_{k}}\right]-x_{j}^{*} \otimes\left[x_{j}, x_{i_{2}}, \ldots, x_{i_{k+1}}\right] \in \operatorname{Im}\left(\tau_{k}^{\prime}\right),
$$

and hence the lemma.

Using the lemmas above, we can reduce the generators of $\operatorname{Coker}\left(\tau_{k}\right)$. We remark that $\operatorname{Im}\left(\tau_{k}^{\prime}\right) \subset \operatorname{Im}\left(\tau_{k}\right)$.

Proposition 3.1. For $k \geq 2$ and $n \geq 4$, $\operatorname{Coker}\left(\tau_{k}\right)$ is generated by $\left(\begin{array}{c}n+k-1 \\ k\end{array}\right)$ elements.

Proof. First, as mentioned above, $H^{*} \otimes_{\mathbf{z}} \mathcal{L}_{n}^{M}(k+1)$ is generated by

$$
\left\{x_{i}^{*} \otimes\left[x_{i_{1}}, \ldots, x_{i_{k+1}}\right] \mid 1 \leq i, i_{1}, \ldots, i_{k+1} \leq n\right\} .
$$

From Lemma 3.2 .

$$
x_{i}^{*} \otimes\left[x_{i_{1}}, \ldots, x_{i_{k+1}}\right] \equiv 0
$$

in $\operatorname{Coker}\left(\tau_{k}\right)$ if $i_{1}, i_{2} \neq i$. For any $x_{i}^{*} \otimes\left[x_{i}, x_{i_{2}}, \ldots, x_{i_{k+1}}\right]$, there exists a $j \in$ $\{1,2, \ldots, n\}$ such that $j \neq i_{2}, i_{k+1}$ since $n \geq 4$. Then

$$
x_{i}^{*} \otimes\left[x_{i}, x_{i_{2}}, \ldots, x_{i_{k+1}}\right]=x_{j}^{*} \otimes\left[x_{j}, x_{i_{2}}, \ldots, x_{i_{k+1}}\right] \in \operatorname{Coker}\left(\tau_{k}\right)
$$

by Lemma 3.4. Hence $\operatorname{Coker}\left(\tau_{k}\right)$ is generated by

$$
\mathfrak{E}=\left\{x_{i}^{*} \otimes\left[x_{i}, x_{i_{2}}, \ldots, x_{i_{k+1}}\right] \mid 1 \leq i, i_{2}, \ldots, i_{k+1} \leq n, \quad i \neq i_{2}, i_{k+1}\right\} .
$$

Furthermore, since $x_{i}^{*} \otimes\left[x_{i}, x_{i_{1}}, \ldots, x_{i_{k}}\right] \in \mathfrak{E}$ does not depend on $i$ by Lemma 3.4 , we can set $s\left(i_{1}, \ldots, i_{k}\right):=x_{i}^{*} \otimes\left[x_{i}, x_{i_{1}}, \ldots, x_{i_{k}}\right]$.

Next, we show

$$
s\left(i_{1}, \ldots, i_{k}\right)=s\left(i_{\lambda(1)}, \ldots, i_{\lambda(k)}\right)
$$


for any $\lambda \in \mathfrak{S}_{k}$. It suffices to show above for each transposition $\lambda=(m m+1)$. If $2 \leq m \leq k-2$, it is trivial by Lemma 3.3 . If $m=k-1$, take $j \in\{1, \ldots, n\}$ such that $j \neq i_{1}, i_{k-1}, i_{k}$. Then

$$
\begin{aligned}
s\left(i_{1}, \ldots, i_{k-1}, i_{k}\right) & =x_{j}^{*} \otimes\left[x_{j}, \ldots, x_{i_{k-1}}, x_{i_{k}}\right]=x_{j}^{*} \otimes\left[x_{j}, \ldots, x_{i_{k}}, x_{i_{k}-1}\right], \\
& =s\left(i_{1}, \ldots, i_{k}, i_{k-1}\right)
\end{aligned}
$$

from Lemma 3.3 Similarly, if $m=1$, take $j \in\{1, \ldots, n\}$ such that $j \neq i_{1}, i_{2}, i_{k}$. Then we obtain $s\left(i_{1}, i_{2}, \ldots, i_{k}\right)=s\left(i_{2}, i_{1}, \ldots, i_{k}\right)$. Hence, we see

$$
\mathfrak{E}^{\prime}=\left\{s\left(i_{1}, \ldots, i_{k}\right) \in \operatorname{Coker}\left(\tau_{k}\right) \mid i_{1} \leq \cdots \leq i_{k}\right\}
$$

generates $\operatorname{Coker}\left(\tau_{k}\right)$. The order of $\mathfrak{E}^{\prime}$ is $\left(\begin{array}{c}n+k-1 \\ k\end{array}\right)$. This completes the proof of Proposition 3.1 ,

3.2. Lower bound on the rank of the cokernel of $\tau_{k}$. In this subsection we give a lower bound on the rank of $\operatorname{Coker}\left(\tau_{k}\right)$ by using the Magnus representation of Aut $F_{n}^{M}$. To do this, we use trace maps introduced by Morita 24] with pioneer and remarkable works. Recently, he showed that there is a symmetric product of $H$ of degree $k$ in the cokernel of the Johnson homomorphism of the automorphism group of a free group using trace maps. Here we apply his method to the case for Aut $F_{n}^{M}$. In order to define the trace maps, we prepare some notation of the associated algebra of the integral group ring. (For basic materials, see [30], Chapter VIII.)

For a group $G$, let $\mathbf{Z}[G]$ be the integral group ring of $G$ over $\mathbf{Z}$. We denote the augmentation map by $\epsilon: \mathbf{Z}[G] \rightarrow \mathbf{Z}$. The kernel $I_{G}$ of $\epsilon$ is called the augmentation ideal. Then the powers of $I_{G}^{i}$ for $i \geq 1$ provide a descending filtration of $\mathbf{Z}[G]$, and the direct sum

$$
\mathfrak{I}_{G}:=\bigoplus_{k \geq 1} I_{G}^{k} / I_{G}^{k+1}
$$

naturally has a graded algebra structure induced from the multiplication of $\mathbf{Z}[G]$. We call $\mathfrak{I}_{G}$ the associated algebra of the group ring $\mathbf{Z}[G]$.

For $G=F_{n}$ a free group of rank $n$, write $I_{n}$ and $\mathfrak{I}_{n}$ for $I_{F_{n}}$ and $\mathfrak{I}_{F_{n}}$ respectively. It is classically well known due to Magnus 23] that each graded quotient $I_{n}^{k} / I_{n}^{k+1}$ is a free abelian group with basis $\left\{\left(x_{i_{1}}-1\right)\left(x_{i_{2}}-1\right) \cdots\left(x_{i_{k}}-1\right) \mid 1 \leq i_{j} \leq n\right\}$, and a map $I_{n}^{k} / I_{n}^{k+1} \rightarrow H^{\otimes k}$ defined by

$$
\left(x_{i_{1}}-1\right)\left(x_{i_{2}}-1\right) \cdots\left(x_{i_{k}}-1\right) \mapsto x_{i_{1}} \otimes x_{i_{2}} \otimes \cdots \otimes x_{i_{k}}
$$

induces an isomorphism from $\mathfrak{I}_{n}$ to the tensor algebra

$$
T(H):=\bigoplus_{k \geq 1} H^{\otimes k}
$$

of $H$ as a graded algebra. We identify $I_{n}^{k} / I_{n}^{k+1}$ with $H^{\otimes k}$ via this isomorphism.

It is also well known that each graded quotient $I_{H}^{k} / I_{H}^{k+1}$ is a free abelian group with basis $\left\{\left(x_{i_{1}}-1\right)\left(x_{i_{2}}-1\right) \cdots\left(x_{i_{k}}-1\right) \mid 1 \leq i_{1} \leq i_{2} \leq \cdots \leq i_{k} \leq n\right\}$, and the associated graded algebra $\mathfrak{I}_{H}$ of $H$ is isomorphic to the symmetric algebra

$$
S(H):=\bigoplus_{k \geq 1} S^{k} H
$$

of $H$ as a graded algebra. (See [30, Chapter VIII, Proposition 6.7.) We also identify $I_{H}^{k} / I_{H}^{k+1}$ with $S^{k} H$. Then a homomorphism $I_{n}^{k} / I_{n}^{k+1} \rightarrow I_{H}^{k} / I_{H}^{k+1}$ induced from the abelianization $\mathfrak{a}: F_{n} \rightarrow H$ is considered as the natural projection $H^{\otimes k} \rightarrow S^{k} H$. 
Now, we define trace maps. For any element $f \in H^{*} \otimes \mathbf{z} \mathcal{L}_{n}^{M}(k+1)$, set

$$
\|f\|:=\left(\frac{\partial\left(x_{i}{ }^{f}\right)}{\partial x_{j}}\right)^{\mathfrak{a}} \in M\left(n, S^{k} H\right)
$$

where we consider any lift of the element

$$
x_{i}^{f} \in \mathcal{L}_{n}^{M}(k+1)=\Gamma_{n}(k+1) /\left(\Gamma_{n}(k+2) \cdot \Gamma_{n}(k+1) \cap F_{n}^{\prime \prime}\right)
$$

to $\Gamma_{n}(k+1)$. Then we define a map $\operatorname{Tr}_{k}^{M}: H^{*} \otimes_{\mathbf{z}} \mathcal{L}_{n}^{M}(k+1) \rightarrow S^{k} H$ by

$$
\operatorname{Tr}_{k}^{M}(f):=\operatorname{trace}(\|f\|) .
$$

It is easily seen that $\operatorname{Tr}_{k}^{M}$ is a $\operatorname{GL}(n, \mathbf{Z})$-equivariant homomorphism. The maps $\operatorname{Tr}_{k}^{M}$ are called the Morita trace maps. We show that $\operatorname{Tr}_{k}^{M}$ is surjective and $\operatorname{Tr}_{k}^{M} \circ \tau_{k}=0$ for $k \geq 2$ and $n \geq 3$. By a direct computation, we obtain

Lemma 3.5. For $f=x_{i}^{*} \otimes\left[x_{i_{1}}, x_{i_{2}}, \ldots, x_{i_{k+1}}\right] \in H^{*} \otimes \mathbf{z} \mathcal{L}_{n}^{M}(k+1)$, we have

$$
\operatorname{Tr}_{k}^{M}(f)=(-1)^{k}\left\{\delta_{i_{1} i} x_{i_{2}} x_{i_{3}} \cdots x_{i_{k+1}}-\delta_{i_{2} i} x_{i_{1}} x_{i_{3}} \cdots x_{i_{k+1}}\right\},
$$

where $\delta_{i j}$ is the Kronecker delta.

Lemma 3.6. For any $k \geq 1$ and $n \geq 2, \operatorname{Tr}_{k}^{M}$ is surjective.

Proof. For any generator $x_{i_{1}} x_{i_{2}} \cdots x_{i_{k}} \in S^{k} H$, we can choose a number $i \in\{1, \ldots, n\}$ such that $i \neq i_{1}$ since $n \geq 2$. Then by Lemma 3.5.

$$
\operatorname{Tr}_{k}^{M}\left(x_{i}^{*} \otimes\left[x_{i}, x_{i_{1}}, \ldots, x_{i_{k}}\right]\right)=(-1)^{k} x_{i_{1}} x_{i_{2}} \cdots x_{i_{k}} .
$$

This shows $\operatorname{Tr}_{k}^{M}$ is surjective.

Before showing $\operatorname{Tr}_{k}^{M} \circ \tau_{k}=0$, we consider a relation between the Magnus representation and the Johnson homomorphism. For each $k \geq 1$, composing the Magnus representation $\operatorname{rep}^{M}$ restricted to $\mathcal{A}_{n}^{M}(k)$ with a homomorphism $\operatorname{GL}(n, \mathbf{Z}[H]) \rightarrow$ $\operatorname{GL}\left(n, \mathbf{Z}[H] / I_{H}^{k+1}\right)$ induced from a natural projection $\mathbf{Z}[H] \rightarrow \mathbf{Z}[H] / I_{H}^{k+1}$, we obtain a homomorphism $\operatorname{rep}_{k}^{M}: \mathcal{A}_{n}^{M}(k) \rightarrow \mathrm{GL}\left(n, \mathbf{Z}[H] / I_{H}^{k+1}\right)$. By the definition of the Magnus representation and the Johnson homomorphism, we obtain

$$
\operatorname{rep}_{k}^{M}(\sigma)=I+\left\|\tau_{k}(\sigma)\right\|
$$

where $I$ denotes the identity matrix. (See also [24].)

Proposition 3.2. For $k \geq 2$ and $n \geq 3, \operatorname{Tr}_{k}^{M}$ vanishes on the image of $\tau_{k}$.

Proof. By Bachmuth [2], we have detorep ${ }^{M}(\sigma)=1$ for any $\sigma \in \mathcal{A}_{n}^{M}(2)$. This shows that

$$
1=\operatorname{det} \circ \operatorname{rep}_{k}^{M}(\sigma)=1+\operatorname{Tr}_{k}^{M}\left(\tau_{k}(\sigma)\right)
$$

for any $\sigma \in \mathcal{A}_{n}^{M}(k)$. Hence $\operatorname{Tr}_{k}^{M}\left(\tau_{k}(\sigma)\right)=0$.

As a corollary, we have

Corollary 3.1. For $k \geq 2$ and $n \geq 3$,

$$
\operatorname{rank}_{\mathbf{Z}}\left(\operatorname{Coker}\left(\tau_{k}\right)\right) \geq\left(\begin{array}{c}
n+k-1 \\
k
\end{array}\right) .
$$


Combining this corollary with Proposition 3.1, we obtain

Theorem 3.1. For $k \geq 2$ and $n \geq 4$,

$$
0 \rightarrow \operatorname{gr}^{k}\left(\mathcal{A}_{n}^{M}\right) \stackrel{\tau_{k}}{\longrightarrow} H^{*} \otimes \mathbf{z} \mathcal{L}_{n}^{M}(k+1) \stackrel{\operatorname{Tr}_{k}^{M}}{\longrightarrow} S^{k} H \rightarrow 0
$$

is a $\mathrm{GL}(n, \mathbf{Z})$-equivariant exact sequence.

Proof. It suffices to show that $\operatorname{Im}\left(\tau_{k}\right)=\operatorname{Ker}\left(\operatorname{Tr}_{k}^{M}\right)$. This immediately follows from Proposition 3.1 and Corollary 3.1. This completes the proof of the theorem.

From (8), we obtain

Corollary 3.2. For $k \geq 2$ and $n \geq 4$,

$$
\operatorname{rank}_{\mathbf{Z}}\left(\operatorname{gr}^{k}\left(\mathcal{A}_{n}^{M}\right)\right)=n k\left(\begin{array}{c}
n+k-1 \\
k+1
\end{array}\right)-\left(\begin{array}{c}
n+k-1 \\
k
\end{array}\right) .
$$

Let $\bar{\nu}_{n, k}: \operatorname{gr}^{k}\left(\mathcal{A}_{n}\right) \rightarrow \operatorname{gr}^{k}\left(\mathcal{A}_{n}^{M}\right)$ be the homomorphism induced from $\nu_{n, k}$. By the argument above, we see that $\operatorname{Im}\left(\tau_{k} \circ \bar{\nu}_{n, k}\right)=\operatorname{Im}\left(\tau_{k}\right)$. Since $\tau_{k}$ is injective, this shows that $\bar{\nu}_{n, k}$ is surjective. Hence

Corollary 3.3. For $k \geq 2$ and $n \geq 4$,

$$
\operatorname{rank}_{\mathbf{Z}}\left(\operatorname{gr}^{k}\left(\mathcal{A}_{n}\right)\right) \geq n k\left(\begin{array}{c}
n+k-1 \\
k+1
\end{array}\right)-\left(\begin{array}{c}
n+k-1 \\
k
\end{array}\right) \text {. }
$$

As mentioned before, in the inequality above, equality does not hold in general, since $\operatorname{rank}_{\mathbf{Z}} \operatorname{gr}^{3}\left(\mathcal{A}_{n}\right)=n\left(3 n^{4}-7 n^{2}-8\right) / 12$, which is not equal to the right hand side of the inequality.

\section{The image of the CuP PRoduct \\ IN THE SECOND COHOMOLOGY GROUP}

In this section, we consider the rational second (co)homology group of $\mathrm{IA}_{n}^{M}$. In particular, we determine the image of the cup product map

$$
\cup_{\mathbf{Q}}^{M}: \Lambda^{2} H^{1}\left(\mathrm{IA}_{n}^{M}, \mathbf{Q}\right) \rightarrow H^{2}\left(\mathrm{IA}_{n}^{M}, \mathbf{Q}\right) .
$$

4.1. A minimal presentation and second cohomology of a group. In this subsection, we consider detecting non-trivial elements of the second cohomology group $H^{2}(G, \mathbf{Z})$ if $G$ has a minimal presentation. For a group $G$, a group extension

$$
1 \rightarrow R \rightarrow F \stackrel{\varphi}{\rightarrow} G \rightarrow 1
$$

is called a minimal presentation of $G$ if $F$ is a free group such that $\varphi$ induces an isomorphism

$$
\varphi_{*}: H_{1}(F, \mathbf{Z}) \rightarrow H_{1}(G, \mathbf{Z}) .
$$

This shows that $R$ is contained in the commutator subgroup $[F, F]$ of $F$. In the following, we assume that $G$ has a minimal presentation defined by (12), and fix it. Furthermore we assume that the rank $m$ of $F$ is finite. We remark that considering the Magnus generators of $\mathrm{IA}_{n}$ and $\mathrm{IA}_{n}^{M}$, we see that each of $\mathrm{IA}_{n}$ and $\mathrm{IA}_{n}^{M}$ has such a minimal presentation. From the cohomological five-term exact sequence of (12), we see

$$
H^{2}(G, \mathbf{Z}) \cong H^{1}(R, \mathbf{Z})^{G} .
$$


Set $\mathcal{L}_{F}(k)=\Gamma_{F}(k) / \Gamma_{F}(k+1)$ for each $k \geq 1$. Then $\mathcal{L}_{F}(k)$ is a free abelian group of rank $r_{m}(k)$ by (3). Let $\left\{R_{k}\right\}_{k \geq 1}$ be a descending filtration defined by $R_{k}:=R \cap \Gamma_{F}(k)$ for each $k \geq 1$. Then $R_{k}=R$ for $k=1$ and 2 . For each $k \geq 1$, let

$$
\varphi_{k}: \mathcal{L}_{F}(k) \rightarrow \mathcal{L}_{G}(k)
$$

be a homomorphism induced from the natural projection $\varphi: F \rightarrow G$. Observing $R_{k} / R_{k+1} \cong\left(R_{k} \Gamma_{F}(k+1)\right) / \Gamma_{F}(k+1)$, we have an exact sequence

$$
0 \rightarrow R_{k} / R_{k+1} \stackrel{\iota_{k}}{\longrightarrow} \mathcal{L}_{F}(k) \stackrel{\varphi_{k}}{\longrightarrow} \mathcal{L}_{G}(k) \rightarrow 0 .
$$

This shows each graded quotient $R_{k} / R_{k+1}$ is a free abelian group.

Set $\bar{R}_{k}:=R / R_{k}$. The natural projection $R \rightarrow \bar{R}_{k}$ induces an injective homomorphism

$$
\psi^{k}: H^{1}\left(\bar{R}_{k}, \mathbf{Z}\right) \rightarrow H^{1}(R, \mathbf{Z}) .
$$

Considering the right action of $F$ on $R$, defined by

$$
r \cdot x:=x^{-1} r x, \quad r \in R, x \in F,
$$

we see $\psi^{k}$ is a $G$-equivariant homomorphism. Hence it induces an injective homomorphism, also denoted by $\psi^{k}$ :

$$
\psi^{k}: H^{1}\left(\bar{R}_{k}, \mathbf{Z}\right)^{G} \rightarrow H^{1}(R, \mathbf{Z})^{G} .
$$

For $k=3, H^{1}\left(\bar{R}_{3}, \mathbf{Z}\right)^{G}=H^{1}\left(\bar{R}_{3}, \mathbf{Z}\right)$, since $G$ acts on $\bar{R}_{3}$ trivially. Here we show that the image of the cup product $\cup: \Lambda^{2} H^{1}(G, \mathbf{Z}) \rightarrow H^{2}(G, \mathbf{Z})$ is contained in $H^{1}\left(\bar{R}_{3}, \mathbf{Z}\right)$.

Lemma 4.1. If $G$ has a minimal presentation as above, the image of the cup product

$$
\cup: \Lambda^{2} H^{1}(G, \mathbf{Z}) \rightarrow H^{2}(G, \mathbf{Z})
$$

is isomorphic to the image of $\iota_{2}^{*}: H^{1}\left(\mathcal{L}_{F}(2), \mathbf{Z}\right) \rightarrow H^{1}\left(\bar{R}_{3}, \mathbf{Z}\right)$.

Proof. First, considering the cohomological five-term exact sequence of

$$
1 \rightarrow \mathcal{A}_{G}^{\prime}(2) \rightarrow G \rightarrow G^{\mathrm{ab}} \rightarrow 1,
$$

we have

$$
0 \rightarrow H^{1}\left(G^{\mathrm{ab}}, \mathbf{Z}\right) \rightarrow H^{1}(G, \mathbf{Z}) \rightarrow H^{1}\left(\mathcal{A}_{G}^{\prime}(2), \mathbf{Z}\right)^{G} \rightarrow H^{2}\left(G^{\mathrm{ab}}, \mathbf{Z}\right) \rightarrow H^{2}(G, \mathbf{Z}) .
$$

Since $H^{1}\left(G^{\mathrm{ab}}, \mathbf{Z}\right) \cong H^{1}(G, \mathbf{Z})$ and $H^{1}\left(\mathcal{A}_{G}^{\prime}(2), \mathbf{Z}\right)^{G}=H^{1}\left(\operatorname{gr}^{2}\left(\mathcal{A}_{G}^{\prime}\right), \mathbf{Z}\right)$, we obtain an exact sequence

$$
0 \rightarrow H^{1}\left(\operatorname{gr}^{2}\left(\mathcal{A}_{G}^{\prime}\right), \mathbf{Z}\right) \rightarrow H^{2}\left(G^{\mathrm{ab}}, \mathbf{Z}\right) \rightarrow H^{2}(G, \mathbf{Z}) .
$$

Since $H_{1}(G, \mathbf{Z})$ is a free abelian group of finite rank, we have a natural isomorphism $H^{2}\left(G^{\mathrm{ab}}, \mathbf{Z}\right) \cong \Lambda^{2} H^{1}(G, \mathbf{Z})$. Then the map $H^{2}\left(G^{\mathrm{ab}}, \mathbf{Z}\right) \rightarrow H^{2}(G, \mathbf{Z})$ is regarded as the cup product $\cup: \Lambda^{2} H^{1}(G, \mathbf{Z}) \rightarrow H^{2}(G, \mathbf{Z})$.

On the other hand, we also consider a five-term exact sequence

$$
\begin{aligned}
0 \rightarrow H^{1}\left(\operatorname{gr}^{2}\left(\mathcal{A}_{G}^{\prime}\right), \mathbf{Z}\right) \rightarrow H^{1}\left(\mathcal{L}_{F}(2), \mathbf{Z}\right) & \rightarrow H^{1}\left(\bar{R}_{3}, \mathbf{Z}\right)^{\mathcal{L}_{F}(2)} \\
& \rightarrow H^{2}\left(\operatorname{gr}^{2}\left(\mathcal{A}_{G}^{\prime}\right), \mathbf{Z}\right) \rightarrow H^{2}\left(\mathcal{L}_{F}(2), \mathbf{Z}\right)
\end{aligned}
$$


of (13) for $k=2$. Since $\mathcal{L}_{F}(2)$ acts on $\bar{R}_{3}$ trivially, we have $H^{1}\left(\bar{R}_{3}, \mathbf{Z}\right)^{\mathcal{L}_{F}(2)}=$ $H^{1}\left(\bar{R}_{3}, \mathbf{Z}\right)$. Then we have a commutative diagram

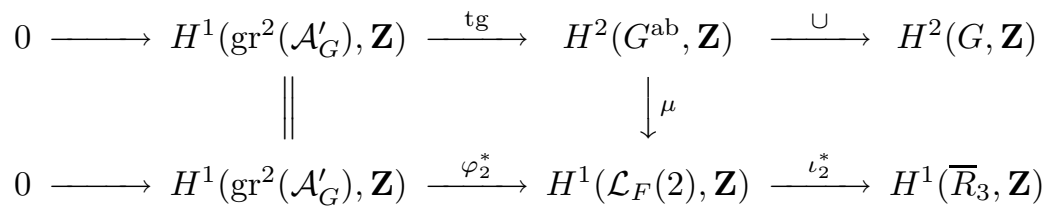

where $\operatorname{tg}$ is the transgression and $\mu$ is a natural isomorphism. Hence we obtain $\operatorname{Im}(\cup) \cong \operatorname{Im}\left(\iota_{2}^{*}\right)$. This completes the proof of Lemma 4.1.

Here we remark that if $\operatorname{gr}^{2}\left(\mathcal{A}_{G}^{\prime}\right)$ is free abelian group, $\operatorname{Im}(\cup)=H^{1}\left(\bar{R}_{3}, \mathbf{Z}\right)$. Furthermore if we consider the rational cup product $\cup_{\mathbf{Q}}: \Lambda^{2} H^{1}(G, \mathbf{Q}) \rightarrow H^{2}(G, \mathbf{Q})$, since $\mathbf{Q}$ is a $\mathbf{Z}$-injective module, the induced homomorphism $\iota_{2}^{*}: H^{1}\left(\mathcal{L}_{F}(2), \mathbf{Q}\right) \rightarrow$ $H^{1}\left(\bar{R}_{3}, \mathbf{Q}\right)$ is surjective. Hence the image of the rational cup product $\cup_{\mathbf{Q}}$ is equal to $H^{1}\left(\bar{R}_{3}, \mathbf{Q}\right)$.

4.2. The image of the rational cup product $\cup_{\mathbf{Q}}^{M}$. In this subsection, we determine the image of the rational cup product

$$
\cup_{\mathbf{Q}}^{M}: \Lambda^{2} H^{1}\left(\mathrm{IA}_{n}^{M}, \mathbf{Q}\right) \rightarrow H^{2}\left(\mathrm{IA}_{n}^{M}, \mathbf{Q}\right) .
$$

First, we should remark that the image of the cup product $\cup_{\mathbf{Q}}: \Lambda^{2} H^{1}\left(\mathrm{IA}_{n}, \mathbf{Q}\right) \rightarrow$ $H^{2}\left(\mathrm{IA}_{n}, \mathbf{Q}\right)$ is completely determined by Pettet 31] who gave the GL $(n, \mathbf{Q})$-irreducible decomposition of it. Here we show that the restriction of $\nu_{n, 1}^{*}: H^{2}\left(\mathrm{IA}_{n}^{M}, \mathbf{Q}\right) \rightarrow$ $H^{2}\left(\operatorname{IA}_{n}, \mathbf{Q}\right)$ to $\operatorname{Im}\left(\cup_{\mathbf{Q}}^{M}\right)$ is an isomorphism onto $\operatorname{Im}\left(\cup_{\mathbf{Q}}\right)$.

To do this, we prepare some notation. Let $F$ be a free group on $K_{i j}$ and $K_{i j k}$ which are corresponding to the Magnus generators of $\mathrm{IA}_{n}$. Namely, $F$ is a free group of rank $n^{2}(n-1) / 2$. Then we have a natural surjective homomorphism $\varphi: F \rightarrow \mathrm{IA}_{n}$ and a minimal presentation

$$
1 \rightarrow R \rightarrow F \stackrel{\varphi}{\longrightarrow} \mathrm{IA}_{n} \rightarrow 1
$$

of $\mathrm{IA}_{n}$, where $R=\operatorname{Ker}(\varphi)$. From a result of Pettet [31, we have

Lemma 4.2. For $n \geq 3, C \bar{R}_{3}$ is a free abelian group of rank

$$
\alpha(n):=\frac{1}{8} n^{2}(n-1)\left(n^{3}-n^{2}-2\right)-\frac{1}{6} n(n+1)\left(2 n^{2}-2 n-3\right) .
$$

Next, we consider the second cohomology groups of $\mathrm{IA}_{n}^{M}$. From now on, we assume $n \geq 4$. We recall that the natural homomorphism $\nu_{n, 1}: \mathrm{IA}_{n} \rightarrow \mathrm{IA}_{n}^{M}$ is surjective, and $\nu_{n, 1}$ induces an isomorphism $\mathrm{IA}_{n}^{\mathrm{ab}} \cong\left(\mathrm{IA}_{n}^{M}\right)^{\mathrm{ab}} \cong H^{*} \otimes \mathbf{z} \Lambda^{2} H$ for $n \geq 4$. Then we have a surjective homomorphism $\varphi^{M}:=\nu_{n, 1} \circ \varphi: F \rightarrow \mathrm{IA}_{n}^{M}$ and a minimal presentation

$$
1 \rightarrow R^{M} \rightarrow F \stackrel{\varphi^{M}}{\longrightarrow} \mathrm{IA}_{n}^{M} \rightarrow 1
$$

of $\operatorname{IA}_{n}^{M}$, where $R^{M}=\operatorname{Ker}(\varphi)$. Observe a sequence

$$
\operatorname{gr}_{\mathbf{Q}}^{2}\left(\mathcal{A}_{n}^{\prime}\right) \rightarrow \operatorname{gr}_{\mathbf{Q}}^{2}\left(\mathcal{A}_{n}^{\prime M}\right) \rightarrow \operatorname{gr}_{\mathbf{Q}}^{2}\left(\mathcal{A}_{n}^{M}\right)
$$


of surjective homomorphisms. Since $\mathcal{A}_{n}(3) / \mathcal{A}_{n}^{\prime}(3)$ is at most a finite abelian group due to Pettet [31, we see

$$
\begin{aligned}
\operatorname{dim}_{\mathbf{Q}}\left(\operatorname{gr}_{\mathbf{Q}}^{2}\left(\mathcal{A}_{n}^{\prime}\right)\right) & =\operatorname{dim}_{\mathbf{Q}}\left(\operatorname{gr}_{\mathbf{Q}}^{2}\left(\mathcal{A}_{n}\right)\right)=\frac{1}{6} n(n+1)\left(2 n^{2}-2 n-3\right) \\
& =\operatorname{dim}_{\mathbf{Q}}\left(\operatorname{gr}_{\mathbf{Q}}^{2}\left(\mathcal{A}_{n}^{M}\right)\right)
\end{aligned}
$$

by (6), and hence $\operatorname{gr}_{\mathbf{Q}}^{2}\left(\mathcal{A}_{n}^{\prime} M\right) \cong \operatorname{gr}_{\mathbf{Q}}^{2}\left(\mathcal{A}_{n}^{M}\right)$. Thus,

Lemma 4.3. For $n \geq 4, C \overline{R_{3}^{M}}$ is a free abelian group of rank $\alpha(n)$.

Proof. Considering the exact sequence of (13) with respect to the minimal presentation (16) for $k=2$ and tensoring it with $\mathbf{Q}$, we obtain

$$
0 \rightarrow\left(\overline{R_{3}^{M}}\right)_{\mathbf{Q}} \rightarrow \mathcal{L}_{F}^{\mathbf{Q}}(2) \stackrel{\varphi_{2, \mathbf{Q}}^{M}}{\longrightarrow} \operatorname{gr}_{\mathbf{Q}}^{2}\left(\mathcal{A}_{n}^{\prime M}\right) \rightarrow 0 .
$$

Hence

$$
\begin{aligned}
\operatorname{rank}_{\mathbf{Z}}\left(\overline{R_{3}^{M}}\right) & =\operatorname{dim}_{\mathbf{Q}}\left(\left(\overline{R_{3}^{M}}\right)_{\mathbf{Q}}\right) \\
& =\operatorname{dim}_{\mathbf{Q}}\left(\mathcal{L}_{F}^{\mathbf{Q}}(2)\right)-\operatorname{dim}_{\mathbf{Q}}\left(\operatorname{gr}_{\mathbf{Q}}^{2}\left(\mathcal{A}_{n}^{M}\right)\right) \\
& =\frac{1}{8} n^{2}(n-1)\left(n^{3}-n^{2}-2\right)-\frac{1}{6} n(n+1)\left(2 n^{2}-2 n-3\right) .
\end{aligned}
$$

Therefore, from the functoriality of the spectral sequence, we obtain commutativity of a diagram

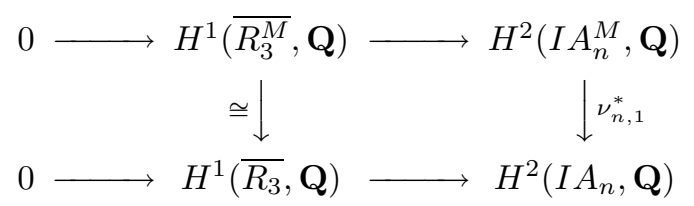

and

Theorem 4.1. For $n \geq 4$, $\nu_{n, 1}^{*}: \operatorname{Im}\left(\cup_{\mathbf{Q}}^{M}\right) \rightarrow \operatorname{Im}\left(\cup_{\mathbf{Q}}\right)$ is an isomorphism.

In Subsection 5.2, we will show the rational cup product $\cup_{\mathbf{Q}}^{M}: \Lambda^{2} H^{1}\left(\mathrm{IA}_{n}^{M}, \mathbf{Q}\right)$ $\rightarrow H^{2}\left(\mathrm{IA}_{n}^{M}, \mathbf{Q}\right)$ is not surjective.

\section{On the kernel of the Magnus Representation of $\mathrm{IA}_{n}$}

In this section, we study the kernel $\mathcal{K}_{n}$ of the Magnus representation of $\mathrm{IA}_{n}$ for $n \geq 4$. Set $\overline{\mathcal{K}}_{n}:=\mathcal{K}_{n} /\left(\mathcal{K}_{n} \cap \mathcal{A}_{n}(4)\right) \subset \operatorname{gr}^{3}\left(\mathcal{A}_{n}\right)$. Since $\left[\mathcal{K}_{n}, \mathcal{K}_{n}\right] \subset \mathcal{A}_{n}(6)$, we see $H_{1}\left(\overline{\mathcal{K}}_{n}, \mathbf{Z}\right)=\overline{\mathcal{K}}_{n}$. Here we determine the $\operatorname{GL}(n, \mathbf{Z})$-module structure of $\overline{\mathcal{K}}_{n}^{\mathbf{Q}}$. As a corollary, we see that the rational cup product $\cup_{\mathbf{Q}}^{M}: \Lambda^{2} H^{1}\left(\mathrm{IA}_{n}^{M}, \mathbf{Q}\right) \rightarrow H^{2}\left(\mathrm{IA}_{n}^{M}, \mathbf{Q}\right)$ is not surjective.

5.1. The irreducible decompositon of $\overline{\mathcal{K}}_{n}^{\mathrm{Q}}$. First, we consider the irreducible decomposition of the target $H_{\mathbf{Q}}^{*} \otimes_{\mathbf{Q}} \mathcal{L}_{n}^{\mathbf{Q}}(4)$ of the rational third Johnson homomorphism $\tau_{3, \mathbf{Q}}$ of Aut $F_{n}$. Let $B$ and $B^{\prime}$ be subsets of $\mathcal{L}_{n}(4)$ consisting of

$$
\left[\left[\left[x_{i}, x_{j}\right], x_{k}\right], x_{l}\right], \quad i>j \leq k \leq l,
$$


and

$$
\begin{aligned}
& {\left[\left[x_{i}, x_{j}\right],\left[x_{k}, x_{l}\right]\right], i>j, k>l, i>k,} \\
& {\left[\left[x_{i}, x_{j}\right],\left[x_{i}, x_{l}\right]\right], \quad i>j, i>l, j>l,}
\end{aligned}
$$

respectively. Then $B \cup B^{\prime}$ forms a basis of $\mathcal{L}_{n}(4)$ due to Hall [15. Let $\mathcal{G}_{n}$ be the $\operatorname{GL}(n, \mathbf{Z})$-equivariant submodule of $\mathcal{L}_{n}(4)$ generated by elements type of $\left[\left[x_{i}, x_{j}\right],\left[x_{k}, x_{l}\right]\right]$ for $1 \leq i, j, k, l \leq n$. Then $B^{\prime}$ is a basis of $\mathcal{G}_{n}$, and the quotient module of $\mathcal{L}_{n}(4)$ by $\mathcal{G}_{n}$ is isomorphic to $\mathcal{L}_{n}^{M}(4)$. Observing that $\mathcal{G}_{n}^{\mathbf{Q}}$ is a $\operatorname{GL}(n, \mathbf{Z})$-equivariant submodule of $\mathcal{L}_{n}^{\mathbf{Q}}(4) \cong H_{\mathbf{Q}}^{[3,1]} \oplus H_{\mathbf{Q}}^{[2,1,1]}$, and $\operatorname{dim}_{\mathbf{Q}}\left(\mathcal{G}_{n}^{\mathbf{Q}}\right)=$ $n\left(n^{2}-1\right)(n+2) / 8$, we see $\mathcal{G}_{n}^{\mathbf{Q}} \cong H_{\mathbf{Q}}^{[2,1,1]}$ and $\mathcal{L}_{n, \mathbf{Q}}^{M}(4) \cong H_{\mathbf{Q}}^{[3,1]}$. Let $D:=\Lambda^{n} H$ be the one-dimensional representation of $\mathrm{GL}(n, \mathbf{Z})$ given by the determinant map. Then considering a natural isomorphism $H_{\mathbf{Q}}^{*} \cong\left(D \otimes_{\mathbf{Q}} \Lambda^{n-1} H_{\mathbf{Q}}\right)$ as a $\mathrm{GL}(n, \mathbf{Z})$ module, and using Pieri's formula (see [13]), we obtain

Lemma 5.1. For $n \geq 4$,

(i) $H_{\mathbf{Q}}^{*} \otimes_{\mathbf{Z}} \mathcal{G}_{n}^{\mathbf{Q}} \cong H_{\mathbf{Q}}^{\left[1^{3}\right]} \oplus H_{\mathbf{Q}}^{[2,1]} \oplus\left(D \otimes \mathbf{Q} H_{\mathbf{Q}}^{\left[3,2^{2}, 1^{n-4}\right]}\right)$,

(ii) $H_{\mathbf{Q}}^{*} \otimes_{\mathbf{Z}} \mathcal{L}_{n, \mathbf{Q}}^{M}(4) \cong H_{\mathbf{Q}}^{[3]} \oplus H_{\mathbf{Q}}^{[2,1]} \oplus\left(D \otimes_{\mathbf{Q}} H_{\mathbf{Q}}^{\left[4,2,1^{n-3}\right]}\right)$.

Now it is clear that $\tau_{3, \mathbf{Q}}\left(\overline{\mathcal{K}}_{n}^{\mathbf{Q}}\right) \subset H_{\mathbf{Q}}^{*} \otimes_{\mathbf{z}} \mathcal{G}_{n}^{\mathbf{Q}}$. On the other hand, in our previous paper [33], we showed that the cokernel of the rational Johnson homomorphism $\tau_{3, \mathbf{Q}}$ is given by $\operatorname{Coker}\left(\tau_{3, \mathbf{Q}}\right)=H_{\mathbf{Q}}^{[3]} \oplus H_{\mathbf{Q}}^{\left[1^{3}\right]}$. Hence we see that $\tau_{3, \mathbf{Q}}\left(\overline{\mathcal{K}}_{n}^{\mathbf{Q}}\right)$ is isomorphic to a submodule of $H_{\mathbf{Q}}^{[2,1]} \oplus\left(D \otimes_{\mathbf{Q}} H_{\mathbf{Q}}^{\left[3,2^{2}, 1^{n-4}\right]}\right)$. In the following, we show $\tau_{3, \mathbf{Q}}\left(\overline{\mathcal{K}}_{n}^{\mathbf{Q}}\right) \cong$ $H_{\mathbf{Q}}^{[2,1]} \oplus\left(D \otimes \mathbf{Q} H_{\mathbf{Q}}^{\left[3,2^{2}, 1^{n-4}\right]}\right)$.

To show this, we prepare some elements of $\mathcal{K}_{n}$. First, for any distinct $p, q, r, s \in$ $\{1,2, \ldots, n\}$ such that $p>q, r$ and $q>r$, set

$$
T(s, p, q, r):=\left[\left[K_{s p}^{-1}, K_{s r}^{-1}\right], K_{s q p}\right] \in \mathrm{IA}_{n} .
$$

Since $T(s, p, q, r)$ satisfies

$$
x_{t} \mapsto \begin{cases}x_{s}\left[\left[x_{p}, x_{q}\right],\left[x_{p}, x_{r}\right]\right], & \text { if } t=s, \\ x_{t}, & \text { if } t \neq s,\end{cases}
$$

$T(s, p, q, r) \in \mathcal{K}_{n}$ and $\tau_{3}(T(s, p, q, r))=x_{s}^{*} \otimes\left[\left[x_{p}, x_{q}\right],\left[x_{p}, x_{r}\right]\right] \in H^{*} \otimes \mathbf{z} \mathcal{G}_{n}$. Next, for any distinct $p, q, r, s \in\{1,2, \ldots, n\}$ such that $p>s$, set

$$
E(s, p, q, r):=\left[\left[K_{s r}, K_{s p q}\right], K_{r s q}\right]\left(K_{r s}^{-1}\left[\left[K_{r s}, K_{s p q}\right]^{-1}, K_{r q}^{-1}\right] K_{r s}\right) \in \mathrm{IA}_{n} .
$$

Then we have

Lemma 5.2. For any $n \geq 4$,

(i) $\tau_{3}(E(s, p, q, r))=x_{s}^{*} \otimes\left[\left[x_{p}, x_{q}\right],\left[x_{s}, x_{q}\right]\right] \in H^{*} \otimes \mathbf{z} \mathcal{G}_{n}$.

(ii) $E(s, p, q, r) \in \mathcal{K}_{n}$.

Proof. Part (i) follows from a direct computation. To prove (ii), we show that $\nu_{n}(E(s, p, q, r)) \in \mathrm{IA}_{n}^{M}$ fix each $x_{t}$ of $F_{n}^{M}$. Here we recall two basic formulae in commutator calculus. For any group $G$ and $x, y, z \in G$,

$$
\begin{aligned}
& {[x y, z]=[x,[y, z]][y, z][x, z],} \\
& {[x, y z]=[x, y][x, z][[z, x], y] .}
\end{aligned}
$$


First, we consider $\nu_{n}\left(\left[\left[K_{s r}, K_{s p q}\right], K_{r s q}\right]\right)$. Since $\left[K_{s r}, K_{s p q}\right] \in \mathrm{IA}_{n}$ satisfies

$$
x_{t} \mapsto \begin{cases}x_{s}\left[\left[x_{q}, x_{p}\right], x_{r}^{-1}\right], & \text { if } t=s, \\ x_{t}, & \text { if } t \neq s,\end{cases}
$$

we see that $\nu_{n}\left(\left[\left[K_{s r}, K_{s p q}\right], K_{r s q}\right]\right)$ fixes $x_{t}$ for $t \neq s, r$, and maps $x_{s}$ and $x_{r}$ as follows:

$$
\begin{aligned}
x_{s} & \stackrel{\nu_{n}\left(\left[K_{s r}, K_{s p q}\right]\right)}{\longrightarrow} x_{s}\left[\left[x_{q}, x_{p}\right], x_{r}^{-1}\right] \stackrel{\nu_{n}\left(K_{r s q}\right)}{\longrightarrow} x_{s}\left[\left[x_{q}, x_{p}\right],\left[x_{q}, x_{s}\right] x_{r}^{-1}\right] \\
& \stackrel{18}{=} x_{s}\left[\left[x_{q}, x_{p}\right],\left[x_{q}, x_{s}\right]\right] \cdot\left[\left[x_{q}, x_{p}\right], x_{r}^{-1}\right] \cdot\left[\left[x_{r}^{-1},\left[x_{q}, x_{p}\right]\right],\left[x_{q}, x_{s}\right]\right] \\
& =x_{s}\left[\left[x_{q}, x_{p}\right], x_{r}^{-1}\right] \stackrel{\nu_{n}\left(\left[K_{s r}, K_{s p q}\right]\right)^{-1}}{\longrightarrow} x_{s} \stackrel{\nu_{n}\left(K_{r s q}\right)^{-1}}{\longrightarrow} x_{s}
\end{aligned}
$$

and

$$
\begin{aligned}
x_{r} & \stackrel{\nu_{n}\left(\left[K_{s r}, K_{s p q}\right]\right)}{\longrightarrow} x_{r} \stackrel{\nu_{n}\left(K_{r s q}\right)}{\longrightarrow} x_{r}\left[x_{s}, x_{q}\right] \stackrel{\nu_{n}\left(\left[K_{s r}, K_{s p q}\right]\right)^{-1}}{\longrightarrow} x_{r}\left[x_{s}\left[x_{r}^{-1},\left[x_{q}, x_{p}\right]\right], x_{q}\right] \\
& \stackrel{17}{=} x_{r}\left[x_{s},\left[\left[x_{r}^{-1},\left[x_{q}, x_{p}\right]\right], x_{q}\right]\right] \cdot\left[\left[x_{r}^{-1},\left[x_{q}, x_{p}\right]\right], x_{q}\right] \cdot\left[x_{s}, x_{q}\right] \\
& =x_{r} \cdot\left(x_{s}\left[\left[x_{r}^{-1},\left[x_{q}, x_{p}\right]\right], x_{q}\right] x_{s}^{-1}\right) \cdot\left[x_{s}, x_{q}\right] \\
& \stackrel{\nu_{n}\left(K_{r s q}\right)^{-1}}{\longrightarrow} x_{r} \cdot\left(x_{s}\left[\left[\left[x_{s}, x_{q}\right] x_{r}^{-1},\left[x_{q}, x_{p}\right]\right], x_{q}\right] x_{s}^{-1}\right) \\
& \stackrel{[17}{=} x_{r} \cdot\left(x_{s}\left[\left[x_{r}^{-1},\left[x_{q}, x_{p}\right]\right], x_{q}\right] x_{s}^{-1}\right) .
\end{aligned}
$$

Next, consider $\left[K_{r s}, K_{s p q}\right]^{ \pm} \in \mathrm{IA}_{n}$. Clearly, these maps fix $x_{t}$ for $t \neq r$, and map $x_{r}$ as follows:

$$
\begin{aligned}
& x_{r} \stackrel{\left[K_{r s}, K_{s p q}\right]}{\longrightarrow} x_{r}\left[x_{r}^{-1},\left[x_{q}, x_{p}\right]\right], \\
& x_{r} \stackrel{\left[K_{r s}, K_{s p q}\right]^{-1}}{\longrightarrow} x_{r}\left[\left[x_{p}, x_{q}\right],\left[\left[x_{q}, x_{p}\right], x_{r}^{-1}\right]\right]\left[\left[x_{q}, x_{p}\right], x_{r}^{-1}\right] .
\end{aligned}
$$

Observing

$$
x_{r}^{\nu_{n}\left(\left[K_{r s}, K_{s p q}\right]^{-1}\right)}=\left[\left[x_{q}, x_{p}\right], x_{r}^{-1}\right] \in F_{n}^{M},
$$

we have

$$
x_{r} \stackrel{\nu_{n}\left(\left[\left[K_{r s}, K_{s p q}\right]^{-1}, K_{r q}^{-1}\right]\right)}{\longrightarrow} x_{r}\left[x_{q},\left[x_{r}^{-1},\left[x_{q}, x_{p}\right]\right]\right]
$$

and

$$
x_{r} \stackrel{\nu_{n}\left(K_{r s}^{-1}\left[\left[K_{r s}, K_{s p q}\right]^{-1}, K_{r q}^{-1}\right] K_{r s}\right)}{\longrightarrow} x_{r} x_{s}\left[x_{q},\left[x_{r}^{-1},\left[x_{q}, x_{p}\right]\right]\right] x_{s}^{-1} .
$$

Therefore we obtain

$$
\begin{gathered}
x_{r} \stackrel{\nu_{n}(E(s, p, q, r))}{\longrightarrow} x_{r}\left(x_{s}\left[x_{q},\left[x_{r}^{-1},\left[x_{q}, x_{p}\right]\right]\right] x_{s}^{-1}\right) \\
\cdot\left(x_{s}\left[\left[\left(x_{s}\left[\left[x_{r}^{-1},\left[x_{q}, x_{p}\right]\right], x_{q}\right] x_{s}^{-1}\right) x_{r}^{-1},\left[x_{q}, x_{p}\right]\right], x_{q}\right] x_{s}^{-1}\right) \\
\stackrel{17}{=} x_{r}\left(x_{s}\left[x_{q},\left[x_{r}^{-1},\left[x_{q}, x_{p}\right]\right]\right] x_{s}^{-1}\right) \cdot\left(x_{s}\left[\left[x_{r}^{-1},\left[x_{q}, x_{p}\right]\right], x_{q}\right] x_{s}^{-1}\right)=x_{r} .
\end{gathered}
$$

This complets the proof of Lemma 5.2 ,

Theorem 5.1. For $n \geq 4, \tau_{3, \mathbf{Q}}\left(\overline{\mathcal{K}}_{n}^{\mathbf{Q}}\right) \cong H_{\mathbf{Q}}^{[2,1]} \oplus\left(D \otimes_{\mathbf{Q}} H_{\mathbf{Q}}^{\left[3,2^{2}, 1^{n-4}\right]}\right)$. 
Proof. Let $\Phi: H^{*} \otimes_{\mathbf{Z}} \mathcal{L}_{n}(4) \rightarrow H^{[2,1]}$ be a map defined by the composition of maps

$$
\Phi: H^{*} \otimes \mathbf{z} \mathcal{L}_{n}(4) \stackrel{\operatorname{id} \otimes \iota_{4}}{\longrightarrow} H^{*} \otimes_{\mathbf{Z}} H^{\otimes 4} \stackrel{C}{\longrightarrow} H^{\otimes 3} \stackrel{f_{[2,1]}}{\longrightarrow} H^{[2,1]}
$$

where $\iota_{4}: \mathcal{L}_{n}(4) \rightarrow H^{\otimes 4}$ is a natural embedding defined by the rule $[X, Y] \mapsto$ $X \otimes Y-Y \otimes X$, a map $C$ is a contraction defined by $C\left(x^{*} \otimes x_{1} \otimes \cdots \otimes x_{4}\right)=$ $x^{*}\left(x_{1}\right) x_{2} \otimes x_{3} \otimes x_{4}$, and $f_{[2,1]}$ is a natural projection. Then $\Phi$ is a $\operatorname{GL}(n, \mathbf{Z})$ equivariant homomorphism.

For the Johnson homomorphism $\tau_{3}$ of Aut, $F_{n}$, we denote by $\tilde{\Phi}$ the restriction of $\Phi$ to $\tau_{3}\left(\overline{\mathcal{K}}_{n}\right)$. By Lemma 5.2 ,

$$
\tilde{\Phi}_{\mathbf{Q}}(E(s, p, q, r))=-2 x_{q} \otimes x_{p} \wedge x_{q} .
$$

Since $\left\{x_{i} \otimes x_{j} \wedge x_{k} \mid j>k \leq i\right\}$ is a basis of $H_{\mathbf{Q}}^{[2,1]}$, we see $\operatorname{Im}(\tilde{\Phi})$ is non-trivial. Hence $\tilde{\Phi}$ is surjective since $H_{\mathbf{Q}}^{[2,1]}$ is a irreducible $\operatorname{GL}(n, \mathbf{Z})$-module. This shows that $\tau_{3, \mathbf{Q}}\left(\overline{\mathcal{K}}_{n}^{\mathbf{Q}}\right)$ contains $H_{\mathbf{Q}}^{[2,1]}$ as an irreducible component.

On the other hand, since $\tilde{\Phi}(T(s, p, q, r))=0$,

$$
T(s, p, q, r) \in \operatorname{Ker}(\tilde{\Phi})=\left(D \otimes_{\mathbf{Q}} H_{\mathbf{Q}}^{\left[3,2^{2}, 1^{n-4}\right]}\right) .
$$

Since $\left(\operatorname{id} \otimes \iota_{4}\right)_{\mathbf{Q}}(T(s, p, q, r)) \neq 0, T(s, p, q, r) \neq 0$ in $\overline{\mathcal{K}}_{n}^{\mathbf{Q}}$. This shows that $\tau_{3, \mathbf{Q}}\left(\overline{\mathcal{K}}_{n}^{\mathbf{Q}}\right)$ also contains $\left(D \otimes \mathbf{Q} H_{\mathbf{Q}}^{\left[3,2^{2}, 1^{n-4}\right]}\right)$ as an irreducible component. Thus we conclude that $\tau_{3, \mathbf{Q}}\left(\overline{\mathcal{K}}_{n}^{\mathbf{Q}}\right) \cong H_{\mathbf{Q}}^{[2,1]} \oplus\left(D \otimes_{\mathbf{Q}} H_{\mathbf{Q}}^{\left[3,2^{2}, 1^{n-4}\right]}\right)$.

Since $\tau_{3, \mathbf{Q}}$ is injective, this shows that

$$
\overline{\mathcal{K}}_{n}^{\mathbf{Q}} \cong H_{\mathbf{Q}}^{[2,1]} \oplus\left(D \otimes \mathbf{Q} H_{\mathbf{Q}}^{\left[3,2^{2}, 1^{n-4}\right]}\right)
$$

and

Corollary 5.1. For $n \geq 4$,

$$
\operatorname{rank}_{\mathbf{Z}}\left(H_{1}\left(\mathcal{K}_{n}, \mathbf{Z}\right)\right) \geq \frac{1}{3} n\left(n^{2}-1\right)+\frac{1}{8} n^{2}(n-1)(n+2)(n-3) .
$$

5.2. Non-surjectivity of the cup product $\cup_{\mathbf{Q}}^{M}$. In this subsection, we also assume $n \geq 4$. Here we show that the rational cup product $\cup_{\mathbf{Q}}^{M}: \Lambda^{2} H^{1}\left(\mathrm{IA}_{n}^{M}, \mathbf{Q}\right) \rightarrow$ $H^{2}\left(\mathrm{IA}_{n}^{M}, \mathbf{Q}\right)$ is not surjective. From the rational five-term exact sequence

$0 \rightarrow H^{1}\left(\mathrm{IA}_{n}^{M}, \mathbf{Q}\right) \rightarrow H^{1}\left(\mathrm{IA}_{n}, \mathbf{Q}\right) \rightarrow H^{1}\left(\mathcal{K}_{n}, \mathbf{Q}\right)^{\mathrm{IA}_{n}} \rightarrow H^{2}\left(\mathrm{IA}_{n}^{M}, \mathbf{Q}\right) \rightarrow H^{2}\left(\mathrm{IA}_{n}, \mathbf{Q}\right)$

of (9), we have an exact sequence

$$
0 \rightarrow H^{1}\left(\mathcal{K}_{n}, \mathbf{Q}\right)^{\mathrm{IA}_{n}} \rightarrow H^{2}\left(\mathrm{IA}_{n}^{M}, \mathbf{Q}\right) \rightarrow H^{2}\left(\mathrm{IA}_{n}, \mathbf{Q}\right) .
$$

By Theorem 4.1, to show the non-surjectivity of the cup product $\cup_{\mathbf{Q}}^{M}$ it suffices to show the non-triviality of $H^{1}\left(\mathcal{K}_{n}, \mathbf{Q}\right)^{\mathrm{IA}_{n}}$.

The natural projection $\mathcal{K}_{n} \rightarrow \overline{\mathcal{K}}_{n}$ induces an injective homomorphism

$$
H^{1}\left(\overline{\mathcal{K}}_{n}, \mathbf{Q}\right) \rightarrow H^{1}\left(\mathcal{K}_{n}, \mathbf{Q}\right)^{\mathrm{IA}_{n}} .
$$

By Theorem 5.1 and the universal coefficients theorem, we see

$$
H^{1}\left(\overline{\mathcal{K}}_{n}, \mathbf{Q}\right) \cong \operatorname{Hom}_{\mathbf{Z}}\left(H_{1}\left(\overline{\mathcal{K}}_{n}, \mathbf{Z}\right), \mathbf{Q}\right) \neq 0 .
$$


Therefore we obtain

Theorem 5.2. For $n \geq 4$, the rational cup product

$$
\cup_{\mathbf{Q}}^{M}: \Lambda^{2} H^{1}\left(\mathrm{IA}_{n}^{M}, \mathbf{Q}\right) \rightarrow H^{2}\left(\mathrm{IA}_{n}^{M}, \mathbf{Q}\right)
$$

is not surjective, and

$$
\operatorname{dim}_{\mathbf{Q}}\left(H^{2}\left(\mathrm{IA}_{n}^{M}, \mathbf{Q}\right)\right) \geq \frac{1}{24} n(n-2)\left(3 n^{4}+3 n^{3}-5 n^{2}-23 n-2\right) .
$$

\section{ACKNOWLEDGMENTS}

The author would like to thank Professor Nariya Kawazumi for valuable advice and useful suggestions. He would also like to express his thanks to the referee for helpful comments and correcting typos and grammatical mistakes. This research is supported by JSPS Research Fellowships for Young Scientists.

\section{REFERENCES}

[1] S. Andreadakis; On the automorphisms of free groups and free nilpotent groups, Proc. London Math. Soc.(3) 15 (1965), 239-268. MR0188307 (32:5746)

[2] S. Bachmuth; Automorphisms of free metabelian groups, Trans. Amer. Math. Soc. 118 (1965), 93-104. MR0180597 (31:4831)

[3] S. Bachmuth; Induced automorphisms of free groups and free metabelian groups, Trans. Amer. Math. Soc. 122 (1966), 1-17. MR0190212 (32:7626)

[4] S. Bachmuth and H. Y. Mochizuki; The non-finite generation of Aut $(G), G$ free metabelian of rank 3, Trans. Amer. Math. Soc. 270 (1982), 693-700. MR645339 (83f:20026)

[5] S. Bachmuth and H. Y. Mochizuki; $\operatorname{Aut}(F) \rightarrow \operatorname{Aut}\left(F / F^{\prime \prime}\right)$ is surjective for free group for rank $\geq 4$, Trans. Amer. Math. Soc. 292, no. 1 (1985), 81-101. MR805954 (87a:20032)

[6] Y. A. Bakhturin, Identities in Lie algebras, Nauka, Moscow 1985; English translation, Identical relations in Lie Algebras, VNU Science Press, Utrecht (1987). MR886063 (88f:17032)

[7] J. S. Birman; Braids, Links, and Mapping Class Groups, Annals of Math. Studies 82 (1974). MR0375281 (51:11477)

[8] K. T. Chen; Integration in free groups, Ann. of Math. 54, no. 1 (1951), 147-162. MR0042414 $(13: 105 \mathrm{c})$

[9] F. Cohen and J. Pakianathan; On Automorphism Groups of Free Groups, and Their Nilpotent Quotients, preprint.

[10] F. Cohen and J. Pakianathan; On subgroups of the automorphism group of a free group and associated graded Lie algebras, preprint.

[11] B. Farb; Automorphisms of $F_{n}$ which act trivially on homology, in preparation.

[12] W. Fulton; Young Tableaux, London Mathematical Society Student Texts 35, Cambridge University Press (1997). MR1464693 (99f:05119)

[13] W. Fulton, J. Harris; Representation Theory, Graduate Texts in Mathematics 129, SpringerVerlag (1991). MR1153249 (93a:20069)

[14] R. Hain; Infinitesimal presentations of the Torelli group, Journal of the American Mathematical Society 10 (1997), 597-651. MR.1431828(97k:14024)

[15] M. Hall; A basis for free Lie rings and higher commutators in free groups, Proc. Amer. Math. Soc. 1 (1950), 575-581. MR0038336(12:388a)

[16] P. J. Hilton and U. Stammbach; A Course in Homological Algebra, Graduate Texts in Mathematics 4, Springer-Verlag, New York (1970). MR1438546 (97k:18001)

[17] D. Johnson; An abelian quotient of the mapping class group, Math. Ann. 249 (1980), 225-242. MR.579103 (82a:57008)

[18] D. Johnson; The structure of the Torelli group III: The abelianization of $\mathcal{I}_{g}$, Topology 24 (1985), 127-144. MR793179 (87a:57016)

[19] N. Kawazumi; Cohomological aspects of Magnus expansions, preprint, arXiv:math.GT/0505497.

[20] S. Krstić, J. McCool; The non-finite presentability in $I A\left(F_{3}\right)$ and $G L_{2}\left(\mathbf{Z}\left[t, t^{-1}\right]\right)$, Invent. Math. 129 (1997), 595-606. MR1465336 (98h:20053) 
[21] R. C. Lyndon, P. E. Schupp; Combinatorial Group Theory, Springer (1977). MR0577064 $(58: 28182)$

[22] W. Magnus; Über n-dimensinale Gittertransformationen, Acta Math. 64 (1935), 353-367. MR.1555401

[23] W. Magnus, A. Karras, D. Solitar; Combinatorial group theory, Interscience Publ., New York (1966). MR2109550 (2005h:20052)

[24] S. Morita; Abelian quotients of subgroups of the mapping class group of surfaces, Duke Mathematical Journal 70 (1993), 699-726. MR1224104 (94d:57003)

[25] S. Morita; Structure of the mapping class groups of surfaces: a survey and a prospect, Geometry and Topology Monographs Vol. 2 (1999), 349-406. MR.1734418 (2000j:57039)

[26] S. Morita; Cohomological structure of the mapping class group and beyond, preprint. MR2264550 (2007j:20079)

[27] J. Nielsen; Die Isomorphismen der allgemeinen unendlichen Gruppe mit zwei Erzeugenden, Math. Ann. 78 (1918), 385-397. MR1511907

[28] J. Nielsen; Die Isomorphismengruppe der freien Gruppen, Math. Ann. 91 (1924), 169-209. MR 1512188

[29] J. Nielsen; Untersuchungen zur Topologie der geschlossenen Zweiseitigen Fläschen, Acta Math. 50 (1927), 189-358. MR.1555256

[30] I. B. S. Passi; Group rings and their augmentation ideals, Lecture Notes in Mathematics 715, Springer-Verlag (1979). MR537126 (80k:20009)

[31] A. Pettet; The Johnson homomorphism and the second cohomology of $I A_{n}$, Algebraic and Geometric Topology 5 (2005), 725-740. MR2153110 (2006j:20050)

[32] C. Reutenauer; Free Lie Algebras, London Mathematical Society Monographs, New Series, no. 7, Oxford University Press (1993). MR1231799 (94j:17002)

[33] T. Satoh; New obstructions for the surjectivity of the Johnson homomorphism of the automorphism group of a free group, Journal of the London Mathematical Society, (2) 74 (2006), 341-360. MR2269583 (2007i:20060)

[34] E. Witt; Treue Darstellung Liescher Ringe, Journal für die Reine und Angewandte Mathematik, 177 (1937), 152-160.

[35] V. M. Zhuravlev; A free Lie algebra as a module over the full linear group, Sbornik Mathematics 187 (1996), 215-236. MR1392842 (97f:20053)

Department of Mathematics, Graduate School of Sciences, Osaka University, 1-16

Machikaneyama, TOYONAKA-CITY, OSAKA 560-0043, JAPAN

E-mail address: takao@math.sci.osaka-u.ac.jp 